\title{
Euler-Poisson systems as action-minimizing paths in the Wasserstein space
}

\author{
W. Gangbo *, T. NGuyen ${ }^{\star \star}$, A. Tudorascu ${ }^{\star \star \star}$
}

\begin{abstract}
This paper uses a variational approach to establish existence of solutions $\left(\sigma_{t}, v_{t}\right)$ for the $1-\mathrm{d}$ Euler-Poisson system by minimizing an action. We assume that the initial and terminal points $\sigma_{0}, \sigma_{T}$ are prescribed in $\mathcal{P}_{2}(\mathbb{R})$, the set of Borel probability measures on the real line, of finite second-order moments. We show existence of a unique minimizer of the action when the time interval $[0, T]$ satisfies $T<\pi$. These solutions conserve the Hamiltonian and they yield a path $t \rightarrow \sigma_{t}$ in $\mathcal{P}_{2}(\mathbb{R})$. When $\sigma_{t}=\delta_{y(t)}$ is a Dirac mass, the Euler-Poisson system reduces to $\ddot{y}+y=0$. The kinetic version of the Euler-Poisson, i.e. the Vlasov-Poisson system was studied in [1] as a Hamiltonian system.
\end{abstract}

\section{Introduction}

Several works are concerned with the Euler-Poisson system and its many variants [11], [17], [20] and [8] (the pressureless case). Some of them have considered the so-called entropy solutions [13], [16]. In this paper, we analyze a different class of solutions for the one-dimensional repulsive EulerPoisson system with constant background. Our goal is to study solutions which are action-minimizing paths with respect to a Lagrangian $L$. This Lagrangian is defined on the tangent bundle to $\mathcal{P}_{2}(\mathbb{R})$ (as defined in [2]).

* WG gratefully acknowledges the support provided by NSF grants DMS-0200267, DMS-03-54729 and DMS-06-00791

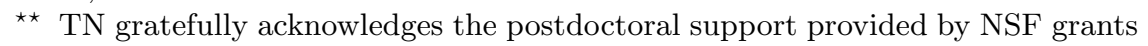
DMS-03-54729 and the School of Mathematics

$\star \star \star$ AT gratefully acknowledges the support provided by the School of Mathematics . Key words: mass transfer, Wasserstein metric. AMS code: 49J40, $82 \mathrm{C} 40$ and 47J25. 
Here, $\mathcal{P}_{2}(\mathbb{R})$ is the space of Borel probability measures on $\mathbb{R}$ with finite second-order moments. When the initial and terminal points of the paths are prescribed, we refer to this problem as the two-point boundary problem. We establish existence of solutions for the two-point boundary problem, along with uniqueness of solutions that are action-minimizer s. Our study is facilitated by a remarkable Eulerian-Lagrangian duality property in the space of $L^{2}$-absolutely continuous curves. This property is a direct consequence of lemma 1. It could also be obtained as a consequence of the more subtle purely analytic result obtained in proposition 6 . These results allow us to pass from Eulerian to Lagrangian coordinates. Here, we require minimal smoothness property in the time variable and no regularity property in the space variable. The result in proposition 6 is also exploited to obtain conservation of the Hamiltonian along solutions in the action-minimizing class.

Let us begin by introducing the commonly known form of the pressureless, repulsive Euler-Poisson system with constant background charge

$$
\left\{\begin{aligned}
\partial_{t} \rho_{t}+\partial_{y}\left(\rho_{t} v_{t}\right) & =0 & & \text { in } \mathbb{R} \times(0, T), \\
\partial_{t}\left(\rho_{t} v_{t}\right)+\partial_{y}\left(\rho_{t} v_{t}^{2}\right) & =-\rho_{t} \partial_{y} \Phi_{t} & & \text { in } \mathbb{R} \times(0, T), \\
-\partial_{y y}^{2} \Phi_{t} & =\rho_{t}-1 & & \text { in } \mathbb{R} .
\end{aligned}\right.
$$

Observe that $y \rightarrow y^{2} / 2-\Phi_{t}(y)$ is a convex function and so, $\partial_{y} \Phi_{t}$ is welldefined except maybe on an at most countable set. Hence, the expression $\rho_{t} \partial_{y} \Phi_{t}$ makes sense since $\partial_{y} \Phi_{t}$ is well-defined $\rho_{t}$-almost everywhere. If, instead, we try to substitute the density $\rho_{t}$ by an arbitrary Borel measure $\sigma_{t}$ in the expression $\rho_{t} \partial_{y} \Phi_{t}$, we are forced to substitute $\partial_{y} \Phi_{t}$ by a function which is defined almost everywhere with respect to $\sigma_{t}$. Thus, to further allow for solutions that are Borel probability measures, we focus on an extension of the momentum equation in (1). Namely we substitute it by

$$
\partial_{t}\left(\sigma_{t} v_{t}\right)+\partial_{y}\left(\sigma_{t} v_{t}^{2}\right)=\sigma_{t}\left[\bar{\gamma}_{t}-\mathbf{i d}\right]
$$

in the distributional sense. Here $\bar{\gamma}_{t}(y)=\sigma_{t}(-\infty, y)+1 / 2 \sigma_{t}\{y\}-1 / 2$. We note that if $\sigma_{t}:=\rho_{t} \mathcal{L}^{1}$, then this formulation and that from (1) coincide. We are going to give a different interpretation of $\bar{\gamma}_{t}$ in the sequel. We arrive to (2) as a natural expression of Newton's second law. To do so, we first need to recall some basic facts from the theory of $L^{2}$-absolutely continuous curves in $\mathcal{P}_{2}(\mathbb{R})$. We shall be quite sketchy; for further details we recommend the comprehensive reference $[2]$. Let us endow $\mathcal{P}_{2}(\mathbb{R})$ with the quadratic Wasserstein metric defined by

$$
W_{2}^{2}(\mu, \nu):=\min _{\gamma} \int_{\mathbb{R}^{2}}|\bar{y}-y|^{2} d \gamma(y, \bar{y}),
$$

where the infimum is taken among all probabilities $\gamma$ on the the product space $\mathbb{R}^{2}$ with marginals $\mu, \nu$. The joint distributions $\gamma$ that realize the minimum are called optimal couplings or optimal plans. Thus, $\left(\mathcal{P}_{2}(\mathbb{R}), W_{2}\right)$ becomes a Polish space on which we define absolutely continuous curves. 
Suppose in general that $(\mathcal{S}$, dist $)$ is a complete metric space. We say that $[0, T] \ni t \rightarrow \sigma_{t} \in \mathcal{S}$ lies in $A C^{2}(0, T ; \mathcal{S})$ provided that there exists $f \in$ $L^{2}(0, T)$ such that $\operatorname{dist}\left(\sigma_{t}, \sigma_{t+h}\right) \leq \int_{t}^{t+h} f(s) d s$ for all $0<t<t+h<T$. We now take $(\mathcal{S}$, dist $)=\left(\mathcal{P}_{2}(\mathbb{R}), W_{2}\right)$ and $\sigma \in A C^{2}\left(0, T ; \mathcal{P}_{2}(\mathbb{R})\right)$. We arrive to the definition of $\bar{\gamma}_{t}$ by first considering $\gamma_{t}$ as the unique optimal coupling between $\nu_{0}:=\left.\mathcal{L}^{1}\right|_{(-1 / 2,1 / 2)}$ and $\sigma_{t}$. Then we let $\bar{\gamma}_{t}$ be the barycentric projection of $\gamma_{t}$ onto its second marginal $\sigma_{t}$. In general, the barycentric projection $\bar{\gamma}_{\mu}: \mathbb{R}^{d} \rightarrow \mathbb{R}^{d}$ of a plan $\gamma \in \mathcal{P}\left(\mathbb{R}^{d} \times \mathbb{R}^{d}\right)$ onto its second marginal $\mu:=\pi_{\#}^{2} \gamma$ is uniquely defined $\mu-$ a.e. by

$$
\bar{\gamma}_{\mu}(y):=\int_{\mathbb{R}^{d}} x d \gamma_{y}(x) \quad \text { for } \mu \text {-a.e. } y \in \mathbb{R}^{d},
$$

where we have disintegrated $\gamma$ as $\gamma=\int_{\mathbb{R}^{d}} \gamma_{y} d \mu(y)$. When $d=1$ one can check that the barycentric projection reduces to $\bar{\gamma}_{\mu}(y)=\mu(-\infty, y)+$ $1 / 2 \mu\{y\}-1 / 2$. To relate back to (1), we make the following observation: if $\sigma_{t}$ vanishes on sets which are at most countable, then $\bar{\gamma}_{t}$ is nothing but the optimal map $\partial_{y} \psi_{t}$ between $\sigma_{t}$ and $\nu_{0}$. Moreover, we have $\partial_{y} \psi_{t}$ is differentiable $\sigma_{t}$-almost everywhere and $\partial_{y y} \psi_{t}=\sigma_{t}$ in the sense of distributions. In this particular case, $\psi_{t}$ and the function $\Phi_{t}$ appearing in (1) are related by $\Phi_{t}(y)=y^{2} / 2-\psi_{t}(y)$.

To arrive to our point of view, we shall briefly discuss a system related to (1) that was recently studied from a similar, yet different perspective. The kinetic version of (1) is the Vlasov-Poisson system

$$
\left\{\begin{aligned}
\partial_{t} f(y, v, t)+v \partial_{y} f(y, v, t) & =-\partial_{y} \phi(y, t) \partial_{v} f(y, v, t) & & \text { in } \mathbb{R} \times \mathbb{R} \times(0, T), \\
\partial_{y y}^{2} \phi(\cdot, t) & =\rho-1 & & \text { in } \mathbb{R} \times(0, T) \\
\rho(y, t) & =\int_{\mathbb{R}} f(y, v, t) d v . & &
\end{aligned}\right.
$$

Indeed, at least at the formal level, if $v_{t}$ is a velocity for $t \rightarrow \rho(\cdot, t)=: \rho_{t}$ and $f(y, w, t)=\rho_{t}(y) \delta_{w-v_{t}(y)}$ (monokinetic solution for Vlasov-Poisson), then $(\rho, v)$ solves $(1)$. Infinite-dimensional Hamiltonian ODE are treated in [1] for Hamiltonians $\mathcal{H}: \mathcal{P}_{2}\left(\mathbb{R}^{d} \times \mathbb{R}^{d}\right) \rightarrow \mathbb{R}$. Set $\mathcal{H}(\nu)=W_{2}^{2}\left(\nu, \nu_{0} \otimes \delta_{0}\right) / 2$ with $\nu_{0}$ being the indicator function of the unit cube $X:=(-1 / 2,1 / 2)^{d}$. One can show that the Hamilton ODE in the sense of [1] becomes the Vlasov-MongeAmpère system introduced in [9]. It appears as an asymptotic approximation for the standard Vlasov-Poisson system describing the evolution of electron clouds in neutralizing uniform media. The two systems are the same if $d=1$. Let us now explain how the Euler-Poisson system (or Euler-Monge-Ampère in multiple dimensions), can also be regarded as a Lagrangian system in a rela ted context. We use the observation already exploited in prior works, valid for all $d$ : if $\nu \in \mathcal{P}_{2}\left(\mathbb{R}^{2 d}\right)$, then

$$
W_{2}^{2}\left(\nu, \nu_{0} \otimes \delta_{0}\right)=\int_{\mathbb{R}^{d}}|v|^{2} d \nu_{2}(v)+W_{2}^{2}\left(\nu_{1}, \nu_{0}\right) .
$$

Here $\nu_{1}$ and $\nu_{2}$ are, respectively, the first and second marginals of $\nu$. Thus, $\mathcal{H}$ defined above does not "see" the full measure $\nu$, only its marginals. Let 
us further restrict our attention to measures $\nu$ of the form $\nu(E)=\mu(\{x \in$ $\left.\left.\mathbb{R}^{d} \mid(x, \zeta(x)) \in E\right\}\right)$ with $\mu \in \mathcal{P}_{2}\left(\mathbb{R}^{d}\right)$ and $\zeta: \mathbb{R}^{d} \rightarrow \mathbb{R}^{d}$ in $L^{2}(\mu)$. We write $\nu=(\mathbf{i d} \times \zeta)_{\#} \mu$, where id stands for the identity map on $\mathbb{R}^{d}$. The tangent space $\mathcal{T}_{\mu} \mathcal{P}_{2}\left(\mathbb{R}^{d}\right)$ is the closure of $\left\{\nabla \varphi \mid \varphi \in C_{c}^{\infty}\left(\mathbb{R}^{d}\right)\right\}$ in $L^{2}(\mu)$ [2], thus it is a separable Hilbert space which we choose to identify with its dual. Therefore, we make no distinction between the tangent and cotangent spaces at $\mu$. We can then restrict ourselves to $\zeta$ in the cotangent bundle at $\mu$. For $\nu$ of the form $(\mathbf{i d} \times \zeta)_{\#} \mu$, the right hand side of (4) gives rise to the Hamiltonian

$$
H(\mu, \zeta):=\frac{1}{2}\|\zeta\|_{L^{2}(\mu)}^{2}+\frac{1}{2} W_{2}^{2}\left(\mu, \nu_{0}\right) .
$$

The associated Lagrangian considered in this paper is

$$
L(\mu, \xi):=\frac{1}{2}\|\xi\|_{L^{2}(\mu)}^{2}-\frac{1}{2} W_{2}^{2}\left(\mu, \nu_{0}\right)=\sup _{\zeta \in \mathcal{T}_{\mu} \mathcal{P}_{2}\left(\mathbb{R}^{d}\right)}\{\langle\zeta, \xi\rangle-H(\mu, \zeta)\} .
$$

It is defined for $\mu \in \mathcal{P}_{2}\left(\mathbb{R}^{d}\right)$ and $\xi \in \mathcal{T}_{\mu} \mathcal{P}_{2}\left(\mathbb{R}^{d}\right)$.

We now take $0<T<\pi$ and consider the action

$$
\mathcal{A}_{T}(\sigma):=\int_{0}^{T} L\left(\sigma_{t}, \mathbf{v}_{t}\right) d t, \quad \sigma \in \mathcal{C}_{T}(\mu, \bar{\mu}) .
$$

Here $\mathcal{C}_{T}(\mu, \bar{\mu})$ denotes the set all paths in $A C^{2}\left(0, T ; \mathcal{P}_{2}\left(\mathbb{R}^{d}\right)\right)$ connecting two given probabilities $\mu, \bar{\mu} \in \mathcal{P}_{2}\left(\mathbb{R}^{d}\right)$. The Borel map $\mathbf{v}: \mathbb{R}^{d} \times(0, T) \rightarrow \mathbb{R}^{d}$ is the velocity of minimal norm associated to $\sigma$. By that we mean first that in the sense of distribution, the continuity equation $\partial_{t} \sigma_{t}+\nabla_{y} \cdot\left(\sigma_{t} \mathbf{v}_{t}\right)=0$ holds in $\mathbb{R}^{d} \times(0, T)$. Secondly, the norm $\left\|\mathbf{v}_{t}\right\|_{L^{2}\left(\sigma_{t}\right)}$ is the metric derivative $\left|\sigma^{\prime}\right|(t)$ for $\mathcal{L}^{1}$-almost every $t \in(0, T)$. As a consequence, $\mathbf{v}_{t} \in \mathcal{T}_{\sigma_{t}} \mathcal{P}_{2}\left(\mathbb{R}^{d}\right)$ for these $t$ (we refer the reader to section 8.3 of [2]). We prove that any critical path for $\mathcal{A}_{T}$ is a solution for the Euler-Poisson system in the sense of distributions. Using a direct method for proving that $\mathcal{A}_{T}$ attains a minimizer which is unique in $\mathcal{C}_{T}(\mu, \bar{\mu})$ seems arduous, mainly because the existence result is complicated by the negative term appearing in $L$. It prevents us from inferring that $\mathcal{A}_{T}$ satisfies any reasonable lower semicontinuity property useful to our purpose. Also, it is not clear that $\mathcal{A}_{T}$ is strictly convex in a sense to be specified. When the space dimension $d=1$, we achieve our goal by switching to the Lagrangian formulation. The key fact here is that for any $\mu, \bar{\mu} \in \mathcal{P}_{2}(\mathbb{R})$, one has $W_{2}(\mu, \bar{\mu})=\left\|M_{\mu}-M_{\bar{\mu}}\right\|_{L^{2}\left(\nu_{0}\right)}$, where $M_{\mu}$ denotes the optimal map such that $M_{\mu \#} \nu_{0}=\mu$. We use this property in lemma 1 to show that $\sigma \in A C^{2}\left(0, T ; \mathcal{P}_{2}(\mathbb{R})\right)$ is equivalent to $M \in H^{1}\left(0, T ; L^{2}\left(\nu_{0}\right)\right)$ and $\left|\sigma^{\prime}\right|(t)=\left|M^{\prime}\right|(t)$ for a.e. $t \in(0, T)$. Here we have set $M_{t}:=M_{\sigma_{t}}$. As a consequence $\left\|v_{t}\right\|_{L^{2}\left(\sigma_{t}\right)}=\left|M^{\prime}\right|(t)$ for a.e. $t \in(0, T)$. In fact, we manage to prove a stronger property which lead to $\left|\sigma^{\prime}\right|(t)=\left|M^{\prime}\right|(t)$. We establish the identity $M_{t}^{\prime}=v_{t} \circ M_{t}$ for a.e. $t \in(0, T)$. Here, $M^{\prime}$ is the functional derivative of $M$ as recalled in (7). Note that here $\sigma_{t}$ may be singular and $v_{t}$ may be completely non-smooth. These facts show that one can switch between the Eulerian and Lagrangian formulations. Before making the latest statement 
more accurate we point out that the remarkable identity $M_{t}^{\prime}=v_{t} \circ M_{t}$ yields conservation of the Hamiltonian $H$ along paths minimizing the action $\mathcal{A}_{T}$. As a consequence of the 1-d setting, one has

$$
W_{2}^{2}\left(\mu, \nu_{0}\right)=\int_{\mathbb{R}} y^{2} d \mu(y)-\frac{1}{2} \int_{\mathbb{R}^{2}}|y-\bar{y}| d \mu(y) d \mu(\bar{y})+\frac{1}{12} .
$$

Then, we mainly focus in this paper on the minimization problem in Lagrangian coordinates. By that we mean analyzing a new action $M \rightarrow$ $\mathcal{Q}(M)+\mathcal{C}(M)$ on $A C^{2}\left(0, T ; L^{2}\left(\nu_{0}\right)\right)$ after the identification of the space $A C^{2}\left(0, T ; L^{2}\left(\nu_{0}\right)\right)$ with the Hilbert space $H^{1}\left(0, T ; L^{2}\left(\nu_{0}\right)\right)$ (as in remark 1.1.3 [2]). It consists of a quadratic term $\mathcal{Q}(M)$ and a convex term $\mathcal{C}(M)$ given by

$$
\begin{gathered}
2 \mathcal{Q}(M)=\int_{0}^{T}\left|M^{\prime}\right|^{2}(t) d t-\int_{0}^{T} d t \int_{X}\left|M_{t}\right|^{2} d \nu_{0}, \\
4 \mathcal{C}(M)=\int_{X^{2}}\left|M_{t} x-M_{t} \bar{x}\right| d x d \bar{x} .
\end{gathered}
$$

By the previous comments, $\mathcal{A}_{T}(\sigma)+T / 24=\mathcal{Q}(M)+\mathcal{C}(M)$. Here, as before, we have assumed that $M_{t} \in L^{2}\left(\nu_{0}\right)$ is the optimal map that pushes $\nu_{0}$ forward to $\sigma_{t}$. Let us prescribe the initial and final maps $\bar{M}_{0}, \bar{M}_{T} \in L^{2}\left(\nu_{0}\right)$. Let $\mathcal{C}_{T}\left(\bar{M}_{0}, \bar{M}_{T}\right)$ be the set of $M \in A C^{2}\left(0, T ; L^{2}\left(\nu_{0}\right)\right)$ such that $M_{0}=$ $\bar{M}_{0}$ and $M_{T}=\bar{M}_{T}$. We prove existence and uniqueness of a minimizer of $\mathcal{Q}(M)+\mathcal{C}(M)$ over $\mathcal{C}_{T}\left(\bar{M}_{0}, \bar{M}_{T}\right)$ if $\bar{M}_{0}, \bar{M}_{T} \in L^{2}(X)$. The Euler-Lagrange equation satisfied by the minimizer $M$ is

$$
M_{t}^{\prime \prime} x+M_{t} x=\frac{1}{2} \int_{X} W(x, \bar{x}, t) d \bar{x} .
$$

Here $W(x, \bar{x}, t) \in \partial .|\cdot|\left(M_{t} x-M_{t}^{1} \bar{x}\right)$ is such that $W(\bar{x}, x, t)=-W(x, \bar{x}, t)$. In fact, since $\mathcal{Q}+\mathcal{C}$ is shown to be strictly convex on $\mathcal{C}_{T}\left(\bar{M}_{0}, \bar{M}_{T}\right),(6)$ characterizes completely its minimizers over $\mathcal{C}_{T}\left(\bar{M}_{0}, \bar{M}_{T}\right)$. We show that if $\bar{M}_{0}$ and $\bar{M}_{T}$ are monotone nondecreasing, then so are $M_{t}$ for $\mathcal{L}^{1}$-almost every $t \in(0, T)$. Note that we are not claiming that $\mathcal{Q}+\mathcal{C}$ is even convex on $H^{1}\left(0, T ; L^{2}\left(\nu_{0}\right)\right)$. We have imposed that $T<\pi$ in order to use Poincaré's inequality in remark 4 and obtain strict convexity of $\mathcal{Q}$ on the smaller set $\mathcal{C}_{T}\left(\bar{M}_{0}, \bar{M}_{T}\right)$.

Due to the lack of differentiability of $|\cdot|$ on the real line, we could not establish (6) by a direct argument. Our strategy was to introduce the function $|\cdot|^{s}$ which is of class $C^{1}(\mathbb{R})$ for $s>1$. We then replace the expression $\mathcal{C}(M)$ by

$$
\mathcal{C}^{s}(M)=\frac{1}{4} \int_{X^{2}}\left|M_{t} x-M_{t} \bar{x}\right|^{s} d x d \bar{x}
$$

It is easy to derive (40) as the Euler-Lagrange equation satisfied by the minimizer $M^{s}$ of $\mathcal{Q}(M)+\mathcal{C}^{s}(M)$ over $\mathcal{C}_{T}\left(\bar{M}_{0}, \bar{M}_{T}\right)$. We then let $s$ tend to 1 to obtain (6).

The characterization of minimizers in (6) is employed to prove a remarkable result: if the two endpoints are averages of $n$ Dirac masses, then so is the 
minimizing path. We refer to this as the closedness principle of $\mathcal{P}_{\cdot n}$, the set of averages of $n$ Dirac masses. Unlike this closedness principle, we provide an example showing the following: if the two endpoints are absolutely continuous with respect to the Lebesgue measure, then the minimizing path is not necessarily so. This is in contrast with the case of minimizing paths for the Lagrangian $\|\xi\|_{L^{2}(\mu)}^{2}$ (i.e. geodesics in the Wasserstein space), which satisfy the property that if the left endpoint is absolutely continuous with respect to the Lebesgue measure, then so are all measures on the geodesic except possibly the right endpoint [18]. Our example also leads to nonuniqueness of energy preserving, entropy solutions for the initial-value problem.

For any $x \in X$, let $S(x, \cdot)$ be the solution of $S^{\prime \prime}(x, t)+S(x, t)=x$, $S(x, 0)=M_{0} x, S(x, T)=M_{T} x$. Then $S(x, t)$ is given by

$$
S(x, t)=\left(M_{0} x-x\right)\left(\cos t-\frac{\cos T}{\sin T} \sin t\right)+\left(M_{T} x-x\right) \frac{\sin t}{\sin T}+x .
$$

Note that depending on $M_{0}$ and $M_{T}$, the function $x \mapsto S(x, t)$ may fail to be monotone nondecreasing and hence cannot be a solution of (6). However, if $S(\cdot, t)$ is increasing for all $t \in(0, T)$, then the path $t \mapsto S(\cdot, t)_{\#} \nu_{0}$ is a minimizing path such that $S(\cdot, t)_{\#} \nu_{0}<<\mathcal{L}^{1}$ for all $t \in[0, T]$. These measures can be computed explicitly. Conversely, if the minimizing path $\sigma$ satisfies $\sigma_{t}<<\mathcal{L}^{1}$ for all $t \in[0, T]$, then we must have $\sigma_{t}:=S(\cdot, t)_{\#} \nu_{0}$.

A formulation similar to (2) appeared in [13], where the initial value problem for a variant of the Euler-Poisson system (attractive, with zero background charge) was studied for $\sigma_{0}<<\mathcal{L}^{1}$ or $\sigma_{0}$ purely atomic. The similarities stop here, as these authors were interested in global solutions satisfying standard entropy conditions. In previous studies such as [8] and [13], one seeks for solutions of the Euler-Poisson system which satisfy the property that when two particles collide, they stick together. Here, we do not impose that condition but obtain that the minimizer of the action $\mathcal{A}_{T}$ satisfies a similar property. By considering the simpler case of the evolution of two particles one discovers that two particles can collide, stick together for some time and then split ways again. But this kind of interaction can occur only once in any time interval of length at most $\pi$ (see Remark (9)).

The analysis described above, however, takes full advantage of the assumption $d=1$ through the isometric identification of $\left(\mathcal{P}_{2}(\mathbb{R}), W_{2}\right)$ with a closed, convex subset of $L^{2}\left(\nu_{0}\right)$. The isometric identification mentioned above fails in higher dimensions. For dimensions $d>1$ we have only been able to check that the Euler-Lagrange equation for the action $\mathcal{A}_{T}$ considered above is the so-called Euler-Monge-Ampère system.

The plan of the paper is as follows: the next section contains some notation and useful preliminaries. This includes general compactness results and a Poincaré-Wirtinger inequality for $A C^{2}(0, T ; \mathcal{S})$ for general complete metric spaces $\mathcal{S}$. It also covers properties of optimal maps in the mass transport problem. In section 3 we prove that Euler-Lagrange equation for the action of $L$ is the Euler-Monge-Ampère system in arbitrary dimensions. Section 4 
is restricted to the one-dimensional case and contains proofs of many of the main results announced above.

Acknowledgements. It is a pleasure to express our gratitude to L. Ambrosio, Y. Brenier, A. Fathi, T. Pacini, A. Swiech and C. Villani for fruitful discussions. We also would like to thank the referee whose many valuable suggestions have helped us to improve the presentation of the paper.

\section{Preliminaries}

In this section we record some notation and definitions used throughout the manuscript. Here $T \in(0, \infty)$ is fixed. We recall well-known facts about the one-dimensional Monge-Kantorovich mass transport theory. A special attention will be devoted to the monotone maps $M: X=(-1 / 2,1 / 2) \rightarrow \mathbb{R}$ which are square integrable. We recall the concept of metric derivative of an absolutely continuous path in a complete metric space. One of the spaces we consider is $\mathcal{P}_{2}(\mathbb{R})$, the set of Borel probability measures on $\mathbb{R}$ with finite second moments, endowed with the Wasserstein distance. Another space which naturally appears is $L^{2}\left(X, \nu_{0}\right)$, the set of square integrable functions on $X$. Here, $\nu_{0}$ is the restriction to $X$ of the one-dimensional Lebesgue measure. We often denote it by $L^{2}\left(\nu_{0}\right)$. A specific subset of it is known to be isometric to $\mathcal{P}_{2}(\mathbb{R})$. We elaborate on that fact in remark 1 (ii) as it will be significantly exploited in this work. Suppose next that $\nu_{0}$ is a Borel probability measure on $\mathbb{R}^{d}$ and let $L^{2}\left(\nu_{0}\right)$ be the set of $M: \mathbb{R}^{d} \rightarrow \mathbb{R}^{d}$ that is square integrable with respect to $\nu_{0}$. Let $\langle\cdot, \cdot\rangle_{\nu_{0}}$ be the standard inner product on $L^{2}\left(\nu_{0}\right)$. We will recall the well-known identification of $L^{2}\left(0, T ; L^{2}\left(\nu_{0}\right)\right)$ and $L^{2}\left(\nu_{0} \times\left.\mathcal{L}^{1}\right|_{(0, T)}\right)$. We consider the space $H^{1}\left(0, T ; L^{2}\left(\nu_{0}\right)\right)$ which consists of $M \in L^{2}\left(0, T ; L^{2}\left(\nu_{0}\right)\right)$ such that its functional derivative $M^{\prime}$ exists in $L^{2}\left(0, T ; L^{2}\left(\nu_{0}\right)\right)$. This means we require

$$
\lim _{h \rightarrow 0}\left\|\frac{M_{t+h}-M_{t}}{h}-M_{t}^{\prime}\right\|_{\nu_{0}}=0
$$

for $\mathcal{L}^{1}$ - a.e. point $t \in(0, T)$, and that $M^{\prime} \in L^{2}\left(0, T ; L^{2}\left(\nu_{0}\right)\right)$. The vector space $H^{1}\left(0, T ; L^{2}\left(\nu_{0}\right)\right)$ is a Hilbert space when endowed with the inner product

$$
\langle M, N\rangle=\int_{0}^{T}\left(\left\langle M_{t}, N_{t}\right\rangle_{\nu_{0}}+\left\langle M_{t}^{\prime}, N_{t}^{\prime}\right\rangle_{\nu_{0}}\right) d t
$$

\subsection{Notation and Definitions}

-We suppose that $T>0$ is a constant. We sometimes give it a specific value such as $T=1$.

- $|\cdot|$ is the euclidean norm on $\mathbb{R}^{d}$ and $\langle\cdot, \cdot\rangle$ is the standard inner product. - $C_{c}^{\infty}\left(\mathbb{R}^{d}\right)$ is the set of functions on $\mathbb{R}^{d}$ which are infinitely differentiable 
and of compact support.

- If $\psi: \mathbb{R}^{d} \rightarrow \mathbb{R} \cup\{ \pm \infty\}, \psi^{*}$ is its Legendre transform.

- id denotes the identity map on $\mathbb{R}^{d}$ for $d \geq 1$.

- As usual, we denote by $\mathcal{L}^{d}$ the Lebesgue measure on $\mathbb{R}^{d}$.

- $X$ denotes the unit cube in $\mathbb{R}^{d}$, centered at the origin. In particular if $d=1$ then $X=(-1 / 2,1 / 2)$. We set $X_{T}:=X \times(0, T)$. Similarly, $X \times X \times(0, T)=$ $X_{T}^{2}$. The measure $\nu_{0}$ is the restriction of $\mathcal{L}^{d}$ to $X$ and so it is a Borel probability measure. We write $\nu_{0}=\left.\mathcal{L}^{d}\right|_{X}$. The product measure of $\nu_{0}$ by $\left.\mathcal{L}^{1}\right|_{(0, T)}$ is the measure on $X_{T}$ denoted by $\nu$. We do not display explicitly its dependence on $T$ since this does not create any confusion in this manuscript. - $\mathcal{P}_{2}\left(\mathbb{R}^{d}\right)$ stands for the set of Borel probability measures $\mu$ on $\mathbb{R}^{d}$ with finite second moments:

$$
\int_{\mathbb{R}^{d}}|y|^{2} d \mu(y)<\infty .
$$

- Given $\mu, \nu \in \mathcal{P}_{2}\left(\mathbb{R}^{d}\right), \Gamma(\mu, \nu)$ is the set of Borel probability measures on $\mathbb{R}^{d} \times \mathbb{R}^{d}$ which have $\mu$ and $\nu$ as their marginals. The Wasserstein distance $W_{2}$ between $\mu$ and $\nu$ is defined by

$$
W_{2}^{2}(\mu, \nu)=\min _{\gamma \in \Gamma(\mu, \nu)} \int_{\mathbb{R}^{2 d}}|x-y|^{2} d \gamma(x, y) .
$$

The set of $\gamma$ where the minimum is achieved is nonempty and is denoted by $\Gamma_{o}(\mu, \nu)$. We refer the reader to [2] chapter 7 for the properties of $W_{2}$ and $\Gamma_{o}(\mu, \nu) .\left(\mathcal{P}_{2}\left(\mathbb{R}^{d}\right), W_{2}\right)$ is a complete and separable metric space. We set $\mathcal{M}:=\mathcal{P}_{2}(\mathbb{R})$.

- If $\mu \in \mathcal{P}_{2}\left(\mathbb{R}^{d}\right), L^{2}(\mu)$ is the set of function $\xi: \mathbb{R}^{d} \rightarrow \mathbb{R}^{d}$ which are $\mu$ measurable and such that $\int_{\mathbb{R}^{d}}|\xi|^{2} d \mu$ is finite. This is a separable Hilbert space for the inner product $\langle\xi, \bar{\xi}\rangle_{\mu}=\int_{\mathbb{R}^{d}}\langle\xi, \bar{\xi}\rangle d \mu$. We denote the associated norm by $\|\cdot\|_{\mu}$. When $m=\left.\mathcal{L}^{1}\right|_{(0, T)}$ to distinguish between the space and time variables, we write $\|\cdot\|_{L^{2}(0, T)}$ for $\|\cdot\|_{m}$.

- If $\mu \in \mathcal{P}_{2}\left(\mathbb{R}^{d}\right)$, we denote by $\mathcal{T}_{\mu} \mathcal{P}_{2}\left(\mathbb{R}^{d}\right)$ the closure of $\left\{\nabla \varphi: \varphi \in C_{c}^{\infty}\left(\mathbb{R}^{d}\right)\right\}$ in $L^{2}(\mu)$. We refer to $\mathcal{T}_{\mu} \mathcal{P}_{2}\left(\mathbb{R}^{d}\right)$ as the tangent space to $\mathcal{P}_{2}\left(\mathbb{R}^{d}\right)$ at $\mu$ (see section 8.5 of [2]). When $d=1$ it is easy to check that $\mathcal{T}_{\mu} \mathcal{P}_{2}(\mathbb{R})=L^{2}(\mu)$. - If $(Z,|\cdot|)$ is a norm space, $L^{2}(0, T ; Z)$ is the set of Borel functions $M$ : $(0, T) \rightarrow Z$ such that $\int_{0}^{T}\left|M_{t}\right|_{Z}^{2} d t<\infty$. Here and throughout this work, we write $M_{t}$ in place of $M(t)$. When $\mu$ is a Borel probability measure on $\mathbb{R}^{d}$ and $Z=L^{2}(\mu)$, we identify $L^{2}\left(0, T ; L^{2}(\mu)\right)$ with $L^{2}\left(\mu \times\left.\mathcal{L}^{1}\right|_{(0, T)}\right)$.

- We also recall that if $M: \mathbb{R}^{d} \rightarrow \mathbb{R}^{d}$ is a Borel map and $\mu \in \mathcal{P}_{2}\left(\mathbb{R}^{d}\right)$ then $M_{\#} \mu$ is the Borel measure defined by

$$
M_{\#} \mu[C]=\mu\left[M^{-1}(C)\right] \quad \text { for all Borel sets } \quad C \subset \mathbb{R}^{d} .
$$

- If $\mu, \nu$ are Borel probability measures on the real line and $\mu$ is atomfree, then it is known that there exists a unique (up to a set of $\mu$-zero measure) optimal map pushing forward $\mu$ to $\nu$. It is called the monotone 
rearrangement and is obtained as $G^{-1} \circ F$, where $F, G$ are the cumulative distribution functions of $\mu$ and $\nu$. We have

$$
G(y)=\nu(-\infty, y] \text { and } G^{-1}(x)=\inf \{y \in \mathbb{R}: G(y) \geq x\} .
$$

Note that $G^{-1}$ is the left-continuous generalized inverse of $G$ (in [21] the right-continuous one is considered). In this work, optimal map on the real line always means left continuous optimal map.

- We denote by Mon the set of monotone nondecreasing functions $M$ : $(-1 / 2,1 / 2) \rightarrow \mathbb{R}$ which are in $L^{2}\left(\nu_{0}\right)$.

- Suppose $(\mathcal{S}$, dist $)$ is a complete metric space and $\sigma:(0, T) \rightarrow \mathcal{S}$. We write $\sigma_{t}$ to denote the value of $\sigma$ at $t: \sigma_{t}:=\sigma(t)$. If there exists $\beta \in L^{2}(0, T)$ such that

$$
\operatorname{dist}\left(\sigma_{t}, \sigma_{s}\right) \leq \int_{s}^{t} \beta(u) d u
$$

for every $s<t$ in $(0, T)$, we say that $\sigma$ is absolutely continuous. We denote by $A C^{2}(0, T ; \mathcal{S})$ the set of $\sigma:(0, T) \rightarrow \mathcal{S}$ that are absolutely continuous.

- Suppose $\sigma \in A C^{2}(0, T ; \mathcal{S})$. Since $\mathcal{S}$ is complete, $\lim _{t \rightarrow 0^{+}} \sigma_{t}$ exists and will be denoted $\sigma_{0}$. Similarly, $\sigma_{T}$ is well-defined. For $\mathcal{L}^{1}$-almost every $t \in(0, T)$

$$
\left|\sigma^{\prime}\right|(t):=\lim _{h \rightarrow 0} \frac{\operatorname{dist}\left(\sigma_{t+h}, \sigma_{t}\right)}{|h|}
$$

exists. If the above limit exists at $t$, we say that $\left|\sigma^{\prime}\right|$ exists at $t$. We have $\left|\sigma^{\prime}\right| \leq \beta$ for every $\beta$ satisfying (10) and

$$
\operatorname{dist}\left(\sigma_{t}, \sigma_{s}\right) \leq \int_{s}^{t}\left|\sigma^{\prime}\right|(u) d u .
$$

The function $\left|\sigma^{\prime}\right|$ is refered to as the metric derivative of $\sigma$. For more details, we refer the reader to section 1.1 of [2]. We denote the $L^{2}$-norm of $\left|\sigma^{\prime}\right|$ on $(0, T)$ by $\left\|\sigma^{\prime}\right\|_{\text {metric }, T}$. In case there is no confusion about the time interval on which we integrate, we simply write $\left\|\sigma^{\prime}\right\|_{\text {metric }}$.

- Suppose $s, \bar{s} \in \mathcal{S}$. We denote by $\mathcal{C}_{T}(s, \bar{s})$ the set of curves $\sigma \in A C^{2}(0, T ; \mathcal{S})$ such that $\sigma_{0}=s$ and $\sigma_{T}=\bar{s}$.

- If $n$ is a integer, $\mathcal{P}$. $n$ is the set of $n$ averages of $n$ Dirac masses in $\mathbb{R}$. When $d=1$, we divide $X=(-1 / 2,1 / 2)$ into $n$ intervals of equal length. Recall that $\nu_{0}$ is the restriction to $X$ of the one-dimensional Lebesgue measure. Suppose $N, \bar{N} \in L^{2}\left(\nu_{0}\right)=: \mathcal{S}$ and are constant on each of these subintervals. We denote by $\mathcal{C}_{T}^{n}(N, \bar{N})$ the set of $M$ in $\mathcal{C}_{T}(N, \bar{N})$ such that for each $t \in(0, T)$, $M_{t}$ is constant on each of these subintervals.

\subsection{Optimal maps}

In this subsection, we recall well-known facts about optimal mass transportation theory. We refer the reader to [2] and [21] for more details.

If $\mu, \bar{\mu} \in \mathcal{P}_{2}(\mathbb{R})$ and $\mu$ vanishes on $(d-1)$-rectifiable sets, then $\Gamma_{o}(\mu, \bar{\mu})$ reduces to a single element $\left\{\gamma_{0}\right\}$. In that case, $\gamma_{0}=(\mathbf{i d} \times \nabla \phi)_{\#} \mu$ for some 
$\phi: \mathbb{R}^{d} \rightarrow \mathbb{R} \cup\{+\infty\}$ convex lower semicontinuous (see [2] chapter 6 and [14]). The map $\nabla \phi$ is the unique (up to a set of $\mu$-zero measure) optimal map such that $\nabla \phi_{\#} \mu=\bar{\mu}$. What we mean is that $\nabla \phi$ is the unique map that minimizes $M \rightarrow \int_{\mathbb{R}^{d}}|\mathbf{i d}-M|^{2} d \mu$ over the set of Borel maps $M$ satisfying $M_{\#} \mu=\bar{\mu}$. When $d=1$ and $X=(-1 / 2,1 / 2) \subset \mathbb{R}, \nu_{0}$ are as above and $\mu=\nu_{0}$ then there is a monotone nondecreasing function $M: X \rightarrow \mathbb{R}$ such that $M_{\#} \nu_{0}=\bar{\mu}$. It is uniquely defined up to a set of $\nu_{0}$-measure and is the optimal map that pushes $\nu_{0}$ forward to $\bar{\mu}$. Since $\int_{\mathbb{R}} y^{2} d \bar{\mu}(y)=\|M\|_{\nu_{0}}^{2}<\infty$ and $M$ is monotone, it achieves only finite values in $X$. Hence, the set of discontinuity points of $M$ is at most countable. The monotone nondecreasing map can be described explicitly. We next write the expression of the one which is left continuous and which will be used throughout this work. For $y \in \mathbb{R}$ and $x \in X$, set

$$
N_{\bar{\mu}}(y)=\bar{\mu}(-\infty, y]-1 / 2, \quad M_{\bar{\mu}}(x)=\inf _{z \in \mathbb{R}}\left\{z: N_{\bar{\mu}}(z) \geq x\right\} .
$$

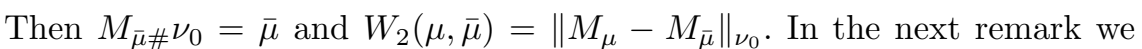
comment on how this well-known identity may be established.

Remark 1. (i) If $M_{0}, M_{T}: X \rightarrow \mathbb{R}^{d}$ are Borel maps and $M_{t \#} \nu_{0}=\sigma_{t}$ for $t=0, T$ then $\gamma:=\left(M_{0} \times M_{T}\right)_{\#} \nu_{0}$ has $\sigma_{0}$ and $\sigma_{T}$ as its marginals and so, $W_{2}^{2}\left(\sigma_{0}, \sigma_{T}\right) \leq \int_{\mathbb{R}^{2}}|y-\bar{y}|^{2} d \gamma(y, \bar{y})=\left\|M_{0}-M_{T}\right\|_{\nu_{0}}^{2}$. Hence, if $M \in A C^{2}\left(0, T ; L^{2}\left(\nu_{0}\right)\right)$ and $\sigma_{t}:=M_{t \#} \nu_{0}$ then $\sigma \in A C^{2}\left(0, T ; \mathcal{P}_{2}\left(\mathbb{R}^{d}\right)\right)$. We then exploit the expression of the metric derivative given in (11) to conclude that $\left|\sigma^{\prime}\right|(t) \leq\left|M^{\prime}\right|(t)$ for $\mathcal{L}^{1}$-almost every $t \in(0, T)$. As a consequence, $\left\|M^{\prime}\right\|_{\text {metric }} \geq\left\|\sigma^{\prime}\right\|_{\text {metric }}$.

(ii) Suppose in addition that $d=1, M_{0}$ and $M_{T}$ are monotone nondecreasing. For each integer number $n$, we choose $\sigma_{0}^{n} \in \mathcal{P}_{2}(\mathbb{R})$ absolutely continuous with respect to $\mathcal{L}^{1}$, of positive density, such that $W_{2}\left(\sigma_{0}^{n}, \sigma_{0}\right) \leq 1 / n$. Let $M_{0}^{n}: X \rightarrow \mathbb{R}$ be monotone increasing satisfying $M_{0 \#}^{n} \nu_{0}=\sigma_{0}^{n}$. The map $M_{0}^{n}$ admits an inverse $N^{n}: \mathbb{R} \rightarrow X$ which is monotone increasing. Since $M_{T} \circ N^{n}$ is monotone nondecreasing and pushes $\sigma_{0}^{n}$ forward to $\sigma_{T}$, we conclude that $W_{2}^{2}\left(\sigma_{0}^{n}, \sigma_{T}\right)=\left\|\mathbf{i d}-M_{T} \circ N^{n}\right\|_{\sigma_{0}^{n}}^{2}$. The last term is checked to be $\left\|M_{0}^{n}-M_{T}\right\|_{\nu_{0}}^{2}$. Letting $n$ tend to $\infty$, we conclude that $W_{2}^{2}\left(\sigma_{0}, \sigma_{T}\right)=\left\|M_{0}-M_{T}\right\|_{\nu_{0}}^{2}$.

A direct consequence of remark 1 and (11) is the following lemma.

Lemma 1. Suppose $\sigma \in L^{2}\left(0, T ; \mathcal{P}_{2}(\mathbb{R})\right), M \in L^{2}\left(0, T ; L^{2}\left(\nu_{0}\right)\right)$ are such that $M_{t}$ is monotone nondecreasing and $M_{t \#} \nu_{0}=\sigma_{t}$. Then we have $\sigma \in$ $A C^{2}\left(0, T ; \mathcal{P}_{2}(\mathbb{R})\right)$ if and only if $M \in A C^{2}\left(0, T ; L^{2}\left(\nu_{0}\right)\right)$. In that case $\left|M^{\prime}\right|(t)$ exists if and only if $\left|\sigma^{\prime}\right|(t)$ exists. Moreover, both functions coincide where they exist.

$$
\text { 2.3. The spaces } A C^{2}\left(0, T ; L^{2}\left(\nu_{0}\right)\right) \text { and } A C^{2}\left(0, T ; \mathcal{P}_{2}\left(\mathbb{R}^{d}\right)\right)
$$

Recall that

$$
X=(-1 / 2,1 / 2)^{d}, \quad X_{T}=X \times(0, T), \quad \nu_{0}=\left.\mathcal{L}^{d}\right|_{X}, \quad \nu:=\nu_{0} \times\left.\mathcal{L}^{1}\right|_{(0, T)} .
$$


One can notice that the next two lemmas are still valid if we replace $X$ by an open subset of $\mathbb{R}^{d}$. The first lemma of this section recalls the standard identification of $L^{2}\left(0, T ; L^{2}\left(\nu_{0}\right)\right)$ and $L^{2}(\nu)$. It allows not to distinguish between these two spaces. Given $N$ as in the lemma below, replacing $N$ by $\tilde{N}$ if necessary, we shall always use the convention that $N \equiv \tilde{N}$. Since the proof of the lemma is standard, it will be skipped.

Lemma 2. If $N \in L^{2}\left(0, T ; L^{2}\left(\nu_{0}\right)\right)$, then there exists $\tilde{N} \in L^{2}(\nu)$ such that

$$
\int_{0}^{T} d t \int_{X} N_{t}(x) \psi(t, x) d x=\int_{X_{T}} \tilde{N}(x, t) \psi(x, t) d x d t
$$

for all $\psi \in L^{2}(\nu)$. Furthermore, $t \rightarrow \tilde{N}(\cdot, t)$ belongs to $L^{2}\left(0, T ; L^{2}\left(\nu_{0}\right)\right)$ and for $\mathcal{L}^{1}$-a.e. $t, \tilde{N}(x, t)=N_{t}(x)$ for $\nu_{0}$-almost every $x \in X$.

The next lemma is also elementary and so, its proof will not be given.

Lemma 3. Suppose that $\{M\} \cup\left\{M^{n}\right\}_{n=1}^{\infty} \subset A C^{2}\left(0, T ; L^{2}\left(\nu_{0}\right)\right)$ satisfies

$$
\left\|M^{n}\right\|_{A C^{2}\left(0, T ; L^{2}\left(\nu_{0}\right)\right)},\|M\|_{A C^{2}\left(0, T ; L^{2}\left(\nu_{0}\right)\right)} \leq C
$$

for a constant $C>0$. Suppose that for each $t \in(0, T),\left\{M_{t}^{n}\right\}_{n=1}^{\infty}$ converges weakly to $M_{t}$ in $L^{2}\left(\nu_{0}\right)$. Then $\left\{M^{n}\right\}_{n=1}^{\infty}$ converges weakly to $M$ in $L^{2}(\nu)$ and $\left\{\left(M^{n}\right)^{\prime}\right\}_{n=1}^{\infty}$ converges weakly to $\left\{M^{\prime}\right\}$ in $L^{2}(\nu)$.

In the remainder of this subsection $d=1$, so that $X=(-1 / 2,1 / 2)$. The purpose of the next two lemmas is to show that if $M \in A C^{2}\left(0, T ; L^{2}\left(\nu_{0}\right)\right)$ and $M_{t}$ is monotone nondecreasing and left continuous for each $t$, then $(t, x) \rightarrow M_{t} x$ is a Borel map. The point is that we do not need to modify $M_{t} x$ on a set of $\mathcal{L}^{2}$-zero measure to obtain a Borel map.

Lemma 4. Let $a<b$ be two real numbers and let $M \in A C^{2}\left(0, T ; L^{2}(a, b)\right)$. Suppose that for each $t$, the function $M_{t}:(a, b) \rightarrow \mathbb{R}$ is monotone, nondecreasing and continuous. Then $(t, x) \rightarrow M_{t} x$ is continuous on $(a, b) \times(0, T)$.

Proof. We skip the proof of this lemma since it is an elementary exercise. We give a hint which is based on the following fact on the class of $C^{1}(a, b)-$ convex functions. Suppose $\left\{f_{n}\right\}_{n=1}^{\infty} \subset C^{1}(a, b)$ are convex, $f \in C^{1}(a, b)$ is convex and $\left\|f_{n}\right\|_{L^{1}(a, b)}$ is bounded. Then $\left\{f_{n}\right\}_{n=1}^{\infty}$ converges weakly in $L_{l o c}^{1}(a, b)$ to $f$ if and only if it converges pointwise in $(a, b)$ to $f$. This is also equivalent to $\left\{f_{n}\right\}_{n=1}^{\infty}$ converges in $C_{l o c}^{0}(a, b)$ to $f$ and $\left\{f_{n}^{\prime}\right\}_{n=1}^{\infty}$ converges pointwise in $(a, b)$ to $f^{\prime}$. Since monotone maps are derivatives of convex functions, one establishes the lemma.

Lemma 5. Let $R \in C_{c}^{1}(\mathbb{R})$ be nonnegative with $\int_{\mathbb{R}} R(y) d y=1$. Suppose $N: X \rightarrow \mathbb{R}$ is a locally bounded function and $N_{-}(x):=\lim _{y \rightarrow x^{-}} N(y)$, $N_{+}(x):=\lim _{y \rightarrow x^{+}} N(y)$ exist for all $x$ in $X$. Set

$$
N^{\epsilon}=R_{\epsilon} * N,
$$


where $R_{\epsilon}(z)=\frac{1}{\epsilon} R\left(\frac{z}{\epsilon}\right)$. Then, for any $x \in\left(-\frac{1}{2}, \frac{1}{2}\right)$, we have

$$
\lim _{\epsilon \rightarrow 0} N^{\epsilon}(x)=\lambda N_{+}(x)+(1-\lambda) N_{-}(x),
$$

where $\lambda=\int_{-\infty}^{0} R(y) d y$. As a consequence, the pointwise limit exists everywhere.

Proof. We have

$$
N^{\epsilon}(x)=\int_{-\infty}^{0} R(z) N(x-\epsilon z) d z+\int_{0}^{\infty} R(z) N(x-\epsilon z) d z \quad \forall x \in X .
$$

Since $N$ is locally bounded in $X$, the dominated convergence theorem yields the conclusion.

Proposition 1. Suppose that $M \in A C^{2}\left(0, T ; L^{2}\left(\nu_{0}\right)\right)$ and for each $t$, the function $M_{t}: X \rightarrow \mathbb{R}$ is monotone, nondecreasing and left continuous. Then $(x, t) \rightarrow M_{t} x$ is Borel on $X_{T}$ as a function of two variables.

Proof. Let $R$ be as in lemma 5 such that $\int_{-\infty}^{0} R(y) d y=0$. Set $M_{t}^{n}=$ $R_{\frac{1}{n}} * M_{t}$. For $0<\delta<1 / 2$, set $X^{\delta}=\left(-\frac{1}{2}+\delta, \frac{1}{2}-\delta\right)$. Then

$$
\left\|M_{t}^{n}-M_{s}^{n}\right\|_{L^{2}\left(X^{\delta}\right)} \leq\left\|M_{t}-M_{s}\right\|_{L^{2}\left(\nu_{0}\right)} \quad \text { for } \quad t, s \in X^{\delta} .
$$

This proves that $M^{n} \in A C^{2}\left(0, T ; L^{2}\left(X^{\delta}\right)\right.$. By Lemma 4 we obtain the map $(x, t) \mapsto M_{t}^{n}(x)$ is continuous on $X_{T}^{\delta}$. By lemma $5 \lim _{n \rightarrow \infty} M_{t}^{n}(x)=M_{t} x$ for each $(x, t) \in X_{T}$. Thus, $M$ is Borel measurable on $X_{T}$ as a pointwise limit of Borel maps.

Observe that the spaces $A C^{2}\left(0, T ; L^{2}\left(\nu_{0}\right)\right)$ and $H^{1}\left(0, T ; L^{2}\left(\nu_{0}\right)\right)$ coincide (see Remark 1.1.3 of [2]). If $M \in H^{1}\left(0, T ; L^{2}\left(\nu_{0}\right)\right)$ we denote by $M^{\prime} \in$ $L^{2}\left(0, T ; L^{2}\left(\nu_{0}\right)\right)$ its functional derivative. It is straightforward to check that $\left|M^{\prime}\right|(t)=\left\|M_{t}^{\prime}\right\|_{\nu_{0}}$ for $\mathcal{L}^{1}$-a.e. $t \in(0, T)$. In the next lemma, we shall view $M$ as a map in $A C^{2}\left(\mathbb{R} ; L^{2}\left(\nu_{0}\right)\right)$ by extending $M_{t}=M_{0+}$ for $t \leq 0$ and $M_{t}=M_{T-}$ for $t \geq T$. Recall that $M^{\prime}$ can be viewed as an element of $L^{2}\left(X_{T}\right)$. We obtain an extension of $M^{\prime}$ to $X \times \mathbb{R}$ which we identify with an element of $L^{2}\left(\nu_{0} \times \mathcal{L}^{1}\right)$.

Lemma 6. Let $M \in A C^{2}\left(0, T ; L^{2}\left(\nu_{0}\right)\right)$ and $M^{\prime}$ be its functional derivative. Then

$$
\lim _{h \rightarrow 0} \int_{X_{T}}\left|\frac{M_{t+h} x-M_{t} x}{h}-M_{t}^{\prime} x\right|^{2} d x d t=0 .
$$

As a consequence, there exist sequences $h_{k}^{+} \rightarrow 0^{+}, h_{k}^{-} \rightarrow 0^{-}$and a measurable subset $A$ of $X \times \mathbb{R}$ such that $\mathcal{L}^{2}((X \times \mathbb{R}) \backslash A)=0$ and

$$
\lim _{k \rightarrow \infty} \frac{M_{t+h_{k}^{+}} x-M_{t} x}{h_{k}^{+}}=\lim _{k \rightarrow \infty} \frac{M_{t+h_{k}^{-}} x-M_{t} x}{h_{k}^{-}}=M_{t}^{\prime} x
$$

for all $(x, t) \in A$. 
Proof. Set $g(t)=\left\|M_{t}^{\prime}\right\|_{\nu_{0}}$ and let $g^{*}(t)$ be the Hardy-Littlewood maximal function given by

$$
g^{*}(t)=\sup _{h}\left|\frac{1}{2 h} \int_{t-h}^{t+h} g(s) d s\right|, \quad t \in \mathbb{R} .
$$

Note that $g \in L^{2}(\mathbb{R})$ and so, $g^{*} \in L^{2}(\mathbb{R})$. Clearly, $\|\left(M_{t+h}-M_{t}\right) / h-$ $M_{t}^{\prime} \|_{\nu_{0}} \leq 2 g^{*}(t)+g(t)$. This, together with (7) and the Lebesgue dominated convergence theorem yields

$$
\lim _{h \rightarrow 0} \int_{0}^{T}\left\|\frac{M_{t+h}-M_{t}}{h}-M_{t}^{\prime}\right\|_{\nu_{0}}^{2} d t=0 .
$$

Fubini's theorem implies (13).

Lemma 7. Assume that $M \in A C^{2}\left(0, T ; L^{2}\left(\nu_{0}\right)\right)$ and for each $t$, the function $M_{t}: X \rightarrow \mathbb{R}$ is monotone nondecreasing. Let $A$ be as in the previous lemma. Suppose $(x, t),(\bar{x}, t) \in A$ and $M_{t} x=M_{t} \bar{x}$. Then $M_{t}^{\prime} x=M_{t}^{\prime} \bar{x}$.

Proof. Without loss of generality, we can assume $x<\bar{x}$. Then

$$
\frac{M_{t+h_{k}^{-}} \bar{x}-M_{t+h_{k}^{-}} x}{h_{k}^{-}} \leq 0 \leq \frac{M_{t+h_{k}^{+}} \bar{x}-M_{t+h_{k}^{+}}}{h_{k}^{+}} .
$$

By (14), this yields $M_{t}^{\prime} x=M_{t}^{\prime} \bar{x}$.

Remark 2. Let $\partial_{t} M$ denote the distributional derivative in time of $(x, t) \rightarrow$ $M_{t} x=M(x, t)$. Then, $M_{t}^{\prime} x=\partial_{t} M(x, t)$ for $\mathcal{L}^{2}$-almost every $(x, t) \in X_{T}$.

Proof. Let $\xi \in C_{c}^{\infty}\left(X_{T}\right)$ be arbitrary. We have

$$
\begin{aligned}
-\int_{X_{T}} \partial_{t} M(x, t) \xi(x, t) d x d t & =\int_{X_{T}} M(x, t) \partial_{t} \xi(x, t) d x d t \\
& =\int_{0}^{T} d t \int_{X} M(x, t) \partial_{t} \xi(x, t) d x \\
& =\lim _{h \rightarrow 0} \int_{0}^{T} d t \int_{X} M(x, t) \frac{\xi(x, t+h)-\xi(x, t)}{h} d x \\
& =\lim _{h \rightarrow 0} \int_{X} d x \int_{0}^{T} M(x, t) \frac{\xi(x, t+h)-\xi(x, t)}{h} d t \\
& =\lim _{h \rightarrow 0} \int_{X} d x \int_{0}^{T} \frac{M(x, t-h)-M(x, t)}{h} \xi(x, t) d t \\
& =\int_{X} d x \int_{0}^{T} M_{t}^{\prime} x \xi(x, t) d t=\int_{X_{T}} M_{t}^{\prime} x \xi(x, t) d x d t .
\end{aligned}
$$

This concludes the proof. 
Recall that (see [2] theorem 8.3.1) if $\sigma \in A C^{2}\left(0, T ; \mathcal{P}_{2}\left(\mathbb{R}^{d}\right)\right)$, then there exists a Borel map $(y, t) \rightarrow \mathbf{v}_{t}(y) \in \mathbb{R}^{d}$ such that

$$
\partial_{t} \sigma_{t}+\nabla_{y} \cdot\left(\sigma_{t} \mathbf{v}_{t}\right)=0 \quad \text { in } \quad \mathbb{R}^{d} \times(0, T)
$$

in the sense of distributions. We call $\mathbf{v}$ a velocity associated to the path $\sigma$. One can choose a velocity associated to the path $\sigma$ so that it is uniquely determined by the following properties: if $\mathbf{w}$ is another velocity associated to the path, then for $\mathcal{L}^{1}$-almost every $t \in(0, T)\left\|\mathbf{v}_{t}\right\|_{L^{2}\left(\sigma_{t}\right)} \leq\left\|\mathbf{w}_{t}\right\|_{L^{2}\left(\sigma_{t}\right)}$ and $\mathbf{v}_{t} \in \mathcal{T}_{\sigma_{t}} \mathcal{P}_{2}\left(\mathbb{R}^{d}\right)$. We refer to $\mathbf{v}_{t}$ as the tangent velocity field at $\sigma_{t}$, or the velocity of minimal norm associated to $\sigma$.

Remark 3. It is known that (see [2] theorem 8.3.1) for $\mathcal{L}^{1}$-almost every $t \in(0, T)$, we have $\left\|\mathbf{v}_{t}\right\|_{L^{2}\left(\sigma_{t}\right)}=\left|\sigma^{\prime}\right|(t)$.

\subsection{Analysis on $A C^{2}(0, T ; \mathcal{S})$}

Throughout this subsection $(\mathcal{S}$, dist $)$ is a complete metric space. We assume the existence of a Hausdorff topology $\tau$ on $\mathcal{S}$, weaker than the metric topology. Also, suppose there exists a distance dist $_{\tau}$ such that on bounded subsets of $(\mathcal{S}$, dist $)$, the topology $\tau$ coincides with the distance topology dist $_{\tau}$. We assume that closed balls of $(\mathcal{S}$, dist) are compact for $\tau$ and that dist is $\tau$-sequentially lower semicontinuous on $B \times B$ whenever $B$ is a closed ball of $(\mathcal{S}$, dist $)$. For instance, when $(\mathcal{S}$, dist $)=\left(L^{2}\left(\nu_{0}\right),\|\cdot\|_{\nu_{0}}\right)$, we choose $\tau$ to be the weak topology. When $\mathcal{S}=\mathcal{P}_{2}\left(\mathbb{R}^{d}\right)$ and dist is the Wasserstein distance, we choose $\tau$ to be the narrow convergence topology (see [2] remark 5.1.1). We extend the Poincaré-Wirtinger inequality from $\mathbb{R}^{d}$ to $\mathcal{S}$.

Proposition 2. Suppose that $\sigma$ belongs to $A C^{2}(0, T ; \mathcal{S})$ and $s_{0} \in \mathcal{S}$. Then

$$
\operatorname{dist}\left(\sigma_{t}, s_{0}\right) \leq \operatorname{dist}\left(\sigma_{0}, s_{0}\right)+\sqrt{t}\left\|\sigma^{\prime}\right\|_{\text {metric }}
$$

and

$$
\pi\left\|\operatorname{dist}\left(\sigma(\cdot), s_{0}\right)\right\|_{L^{2}(0, T)} \leq T\left\|\sigma^{\prime}\right\|_{m e t r i c}+\sqrt{T} \mathcal{W}_{\mathcal{S}}\left(\sigma_{0}, \sigma_{T} ; s_{0}\right)
$$

where $\mathcal{W}_{\mathcal{S}}\left(\sigma_{0}, \sigma_{T} ; \nu_{o}\right)$ is defined below. We also have

$$
\pi\left\|\operatorname{dist}\left(\sigma(\cdot), s_{0}\right)\right\|_{L^{2}(0, T)} \leq 2 T\left\|\sigma^{\prime}\right\|_{\text {metric }}+\pi \sqrt{T} \operatorname{dist}\left(\sigma_{T}, s_{0}\right) .
$$

Here, $\mathcal{W}_{\mathcal{S}}\left(\sigma_{0}, \sigma_{T} ; s_{0}\right)=\pi\left(\operatorname{dist}\left(\sigma_{0}, s_{0}\right) \operatorname{dist}\left(\sigma_{T}, s_{0}\right)+\frac{1}{3} \operatorname{dist}^{2}\left(\sigma_{0}, \sigma_{T}\right)\right)^{1 / 2}+$ $\operatorname{dist}\left(\sigma_{0}, \sigma_{T}\right)$.

Proof. Set $u(t)=\operatorname{dist}\left(\sigma_{t}, s_{0}\right)$. Then for all $h>0$ and $t \in(0, T)$

$$
(|u(t+h)-u(t)|) / h \leq \operatorname{dist}\left(\sigma(t+h), \sigma_{t}\right) / h .
$$


This proves that $u \in H^{1}(0, T)$ and $\left|u^{\prime}(t)\right| \leq\left|\sigma^{\prime}\right|(t)$ for $\mathcal{L}^{1}$-almost every $t \in(0, T)$. We exploit this and the identity $u(t)=u(0)+\int_{0}^{t} u^{\prime}(s) d s$ to obtain (15). Set

$$
w(t):=u(t)-u(0)+(t / T)(u(0)-u(T)), \tilde{w}(t):=\left|\sigma^{\prime}\right|(t)+\frac{1}{T} \operatorname{dist}\left(\sigma_{0}, \sigma_{t}\right) .
$$

Then $w \in H_{0}^{1}(0, T)$ and so, by the standard Poincaré inequality, since we have $\left|w^{\prime}\right| \leq \tilde{w}$

$$
(\pi / T)\|w\| \leq\left\|w^{\prime}\right\| \leq\|\tilde{w}\| \leq\left\|\sigma^{\prime}\right\|_{m e t r i c}+\frac{1}{\sqrt{T}} \operatorname{dist}\left(\sigma_{0}, \sigma_{T}\right),
$$

where the norm $\|\cdot\|:=\|\cdot\|_{L^{2}(0, T)}$ This, together with the fact that

$$
\int_{0}^{T}((1-t / T) a+(t / T) b)^{2} d t=(T / 3)(b-a)^{2}+T a b,
$$

yields (16). We use that $\pi\|u-u(T)\|_{L^{2}(0, T)} \leq 2 T\left\|u^{\prime}\right\|_{L^{2}(0, T)}$ to obtain (17).

Proposition 3. Suppose that $\sigma:[0, T] \rightarrow \mathcal{S},\left\{\sigma^{n}\right\}_{n=1}^{\infty} \subset A C^{2}(0, T ; \mathcal{S})$ and $\left\{\sigma_{t}^{n}\right\}_{n=1}^{\infty}$ converges to $\sigma_{t}$ in $\left(\mathcal{S}\right.$, dist $\left._{\tau}\right)$ for every $t \in(0, T)$. If $\left\{\sigma_{0}^{n}\right\}_{n=1}^{\infty}$ is bounded in $(\mathcal{S}$, dist $)$, then $\sigma \in A C^{2}(0, T ; \mathcal{S})$ and

$$
\liminf _{n \rightarrow \infty}\left\|\left(\sigma^{n}\right)^{\prime}\right\|_{\text {metric }} \geq\left\|\sigma^{\prime}\right\|_{\text {metric }} .
$$

Proof. Without loss of generality, we assume the left hand side of (18) is finite and let $\left\{n_{k}\right\}_{k=1}^{\infty} \subset \mathbf{N}$ be such that

$$
C_{0}=\lim _{k \rightarrow+\infty}\left\|\left(\sigma^{n_{k}}\right)^{\prime}\right\|_{\text {metric }}=\liminf _{n \rightarrow \infty}\left\|\left(\sigma^{n}\right)^{\prime}\right\|_{\text {metric }} .
$$

The sequence $\left\{\left|\left(\sigma^{n_{k}}\right)^{\prime}\right|\right\}_{k=1}^{\infty}$ is bounded in $L^{2}(0, T)$ and so, it admits a subsequence (not relabelled) which converges weakly to some $\alpha$ in $L^{2}(0, T)$. Since $\left\{\sigma_{0}^{n}\right\}_{n=1}^{\infty}$ is bounded in $\left(\mathcal{S}\right.$, dist), (12) yields that $\left\{\sigma_{t}^{n}\right\}_{n=1}^{\infty}$ is bounded in $(\mathcal{S}$, dist) for each $t \in(0, T)$. We use (12) again and the fact that dist is dist $_{\tau}$-lower semicontinuous on dist-bounded sets to obtain

$$
\operatorname{dist}\left(\sigma_{t}, \sigma_{s}\right) \leq \liminf _{k \rightarrow+\infty} \operatorname{dist}\left(\sigma_{t}^{n_{k}}, \sigma_{s}^{n_{k}}\right) \leq \liminf _{k \rightarrow+\infty} \int_{s}^{t}\left|\left(\sigma^{n_{k}}\right)^{\prime}\right|(u) d u=\int_{s}^{t} \alpha(u) d u
$$

for every $0<s \leq t<T$. This proves that $\sigma \in A C^{2}(0, T ; \mathcal{S})$. By the minimality property of $\left|\sigma^{\prime}\right|$, (19) yields $\left|\sigma^{\prime}\right| \leq \alpha$. This, together with the lower semicontinuity of $\|\cdot\|_{L^{2}(0, T)}$ gives that

$$
\liminf _{k \rightarrow+\infty}\left\|\left(\sigma^{n_{k}}\right)^{\prime}\right\|_{\text {metric }} \geq\|\alpha\|_{L^{2}(0, T)} \geq\left\|\sigma^{\prime}\right\|_{\text {metric }}
$$

This concludes the proof.

The following proposition will be used often in this work. It appears as proposition 3.3.1 in [2]. 
Proposition 4. Suppose $\left\{\sigma_{n}\right\}_{n=1}^{\infty}$ is a sequence in $A C^{2}(a, b ; \mathcal{S})$ satisfying

$$
M:=\sup _{n}\left\|\left(\sigma_{n}\right)^{\prime}\right\|_{m e t r i c}, \quad \bar{M}:=\sup _{n} \operatorname{dist}\left(\sigma_{n}(0), \nu_{0}\right)<\infty
$$

for some $\nu_{0} \in \mathcal{S}$. Then, there exist $\sigma \in A C^{2}(a, b ; \mathcal{S})$ and a sequence of positive integers $\left\{n_{k}\right\}_{k=1}^{\infty}$ (independent of $t$ ) such that $\left\{\sigma_{n_{k}}(t)\right\}_{k=1}^{\infty}$ converges to $\sigma_{t}$ in $\left(\mathcal{S}\right.$, dist $\left._{\tau}\right)$ for every $t \in(a, b)$.

\section{Actions on $A C^{2}\left(0, T ; \mathcal{P}_{2}\left(\mathbb{R}^{d}\right)\right)$ and $A C^{2}\left(0, T ; L^{2}\left(\nu_{0}\right)\right)$}

In this section we assume that $T \in(0, \pi)$. This condition is not needed in subsection 3.1 but it is useful in the remaining subsections.

\subsection{Euler-Monge-Ampère systems as minimizers of an action}

On $A C^{2}\left(0, T ; \mathcal{P}_{2}\left(\mathbb{R}^{d}\right)\right)$ we consider the action

$$
\mathcal{A}_{T}(\sigma):=\frac{1}{2}\left(\left\|\sigma^{\prime}\right\|_{\text {metric }}^{2}-\left\|W_{2}^{2}\left(\sigma(\cdot), \nu_{0}\right)\right\|_{L^{2}(0, T)}^{2}\right) .
$$

In the remainder of this subsection, we fix $\sigma \in A C^{2}\left(0, T ; \mathcal{P}_{2}\left(\mathbb{R}^{d}\right)\right)$ and $\xi \in C_{c}^{\infty}\left((0, T) \times \mathbb{R}^{d} ; \mathbb{R}^{d}\right)$. We define $\sigma_{t}^{s}=(\mathbf{i d}+s \xi(t, \cdot))_{\#} \sigma_{t}$, where we recall that $\operatorname{id}(y) \equiv y$. Let $M \in A C^{2}\left(0, T ; L^{2}\left(\nu_{0}\right)\right)$ be such that $M_{t \#} \nu_{0}=\sigma_{t}$ and $M_{t}$ is monotone nondecreasing.

Lemma 8. For $s \in \mathbb{R}, \sigma^{s} \in A C^{2}\left(0, T ; \mathcal{P}_{2}\left(\mathbb{R}^{d}\right)\right)$ and, if $s$ is small enough,

$$
\left|\left(\sigma^{s}\right)^{\prime}\right|^{2}(t)-\left|\sigma^{\prime}\right|^{2}(t) \leq C_{t}^{2} s^{2}+2 s \int_{\mathbb{R}^{d}}\left\langle\mathbf{v}_{t}, \partial_{t} \xi_{t}+\nabla_{y} \xi_{t} \mathbf{v}_{t}\right\rangle d \sigma_{t}
$$

for $\mathcal{L}^{1}$-a.e. $t \in(0, T)$, where $C_{t}=\left\|\partial_{t} \xi+\nabla \xi_{t} \cdot \mathbf{v}_{t}\right\|_{L^{2}\left(\sigma_{t}\right)}$.

Proof. Set $M_{t}=\mathbf{i d}+s \xi_{t}$. For arbitrary $F \in C_{c}^{\infty}\left(\mathbb{R}^{d}\right)$, it is easy to see that the derivative of $t \rightarrow \int_{\mathbb{R}^{d}} F d \sigma_{t}^{s}=\int_{\mathbb{R}^{d}} F\left(M_{t}\right) d \sigma_{t}$ with respect to $t$ is $\int_{\mathbb{R}^{d}}\left\langle\nabla F, \mathbf{v}_{t}^{s}\right\rangle d \sigma_{t}^{s}$, where

$$
\mathbf{v}_{t}^{s}=\left[\mathbf{v}_{t}+s\left(\partial_{t} \xi+\nabla \xi_{t} \cdot \mathbf{v}_{t}\right)\right] \circ M_{t}^{-1} .
$$

Hence, $\mathbf{v}^{s}$ is a velocity for the $\sigma^{s}$ (we also refer the reader to theorem 2.1 of [3] for more details). By the fact that $\left|\left(\sigma^{s}\right)^{\prime}\right|(t)$ is the minimal norm of admissible velocities of $\sigma^{s}$, we conclude that for $\mathcal{L}^{1}$-almost every $t \in(0, T)$

$$
\left|\left(\sigma^{s}\right)^{\prime}\right|(t) \leq\left\|\mathbf{v}_{t}^{s}\right\|_{L^{2}\left(\sigma_{t}^{s}\right)} .
$$

From that, it is apparent that the lemma holds. 
Lemma 9. Let $\mu \in \mathcal{P}_{2}\left(\mathbb{R}^{d}\right), \eta \in C_{c}^{\infty}\left(\mathbb{R}^{d}\right)$ and set $\mu^{s}=(\mathbf{i d}+s \eta)_{\#} \mu$. Let $\varphi$ be a lower semicontinuous convex function such that $(\nabla \varphi)_{\#} \nu_{0}=\mu$. Then,

$$
\lim _{s \rightarrow 0} \frac{W_{2}^{2}\left(\mu^{s}, \nu_{0}\right)-W_{2}^{2}\left(\mu, \nu_{0}\right)}{s}=2 \int_{\mathbb{R}^{d}}\langle\mathbf{i d}-\bar{\gamma}, \eta\rangle d \mu .
$$

Here $\gamma=(\mathbf{i d} \times \nabla \varphi)_{\#} \nu_{0}$ and $\bar{\gamma} \in \mathcal{T}_{\mu} \mathcal{P}_{2}\left(\mathbb{R}^{d}\right)$ is the barycentric projection of $\gamma$ onto $\mu$ (see (3) in the introduction).

Proof. We have $W_{2}\left(\mu^{s}, \mu\right) \leq s\|\eta\|_{\mu}$ and so, by the triangle inequality, $W_{2}\left(\mu^{s}, \nu_{0}\right)$ tends to $W_{2}\left(\mu, \nu_{0}\right)$ as $s$ tends to 0 . Set $T_{s}=\mathbf{i d}+s \eta$ and let $\varphi_{s}$ be the convex function satisfying $\left(\nabla \varphi_{s}\right)_{\# \nu_{0}}=\mu^{s}$. Observe that $\Gamma_{o}\left(\nu_{0}, \mu\right)$ has only one element. Hence, it is obvious that $\left\{\nabla \varphi_{s}\right\}$ converges weakly in $L^{2}\left(\nu_{0}\right)$ to $\nabla \varphi$ as $s$ tends to 0 (see for instance [1] lemma 3.3). Since $W_{2}\left(\mu^{s}, \nu_{0}\right)^{2}=\left\|\nabla \varphi_{s}-\mathbf{i d}\right\|_{\nu_{0}}^{2}$ and $W_{2}^{2}\left(\mu, \nu_{0}\right)^{2}=\|\nabla \varphi-\mathbf{i d}\|_{\nu_{0}}^{2}$ we conclude that $\left\{\nabla \varphi_{s}\right\}$ converges strongly in $L^{2}\left(\nu_{0}\right)$ to $\nabla \varphi$ as $s$ tends to 0 . Note that $\left(T_{s} \operatorname{circ} \nabla \varphi\right)_{\# \nu_{0}}=\mu^{s}$ and so,

$$
\begin{array}{r}
W_{2}^{2}\left(\mu^{s}, \nu_{0}\right)-W_{2}^{2}\left(\mu, \nu_{0}\right) \leq \int_{X}\left|\mathbf{i d}-T_{s} \circ \nabla \varphi\right|^{2} d \nu_{0}-\int_{X}|\mathbf{i d}-\nabla \varphi|^{2} d \nu_{0} \\
=2 s \int_{X}\langle\nabla \varphi-\mathbf{i d}, \eta \circ \nabla \varphi\rangle d \nu_{0}+s^{2} \int_{X}|\xi \circ \nabla \varphi|^{2} d \nu_{0} .
\end{array}
$$

On the other hand, $T_{s}^{-1}$ exists and $\left(T_{s}^{-1} \circ \nabla \varphi_{s}\right)_{\# \nu_{0}}=\mu^{s}$ if $s$ is sufficiently small. Thus,

$$
\begin{array}{r}
W_{2}^{2}\left(\mu, \nu_{0}\right)-W_{2}^{2}\left(\mu^{s}, \nu_{0}\right) \leq \int_{X}\left|\mathbf{i d}-T_{s}^{-1} \circ \nabla \varphi_{s}\right|^{2} d \nu_{0}-\int_{X}\left|\mathbf{i d}-\nabla \varphi_{s}\right|^{2} d \nu_{0} \\
=2 s \int_{X}\left\langle\frac{\left(\mathbf{i d}-T_{s}^{-1}\right) \circ \nabla \varphi_{s}}{s}, \mathbf{i d}-\frac{\left(\mathbf{i d}+T_{s}^{-1}\right) \circ \nabla \varphi_{s}}{2}\right\rangle d \nu_{0}=: 2 s A_{s} .
\end{array}
$$

It is easy to check that $\left|T_{s}^{-1}(y)-y+s \xi(y, t)\right| \leq C s^{2}$ for a constant $C$ independent of $s$ and $y$ and so,

$$
A_{s}=\frac{s^{2}}{2}\|\eta\|_{\nu_{0}}^{2}+\int_{X}\left\langle\mathbf{i d}-\nabla \varphi_{s}, \eta \circ \nabla \varphi_{s}\right\rangle d \nu_{0}+0\left(s^{2}\right) .
$$

We combine (22), (23), (24) and use the fact that $\left\{\nabla \varphi_{s}\right\}$ converges strongly in $L^{2}\left(\nu_{0}\right)$ to $\nabla \varphi$ as $s$ tends to 0 to conclude the proof. The assumption $\gamma \in \Gamma_{o}\left(\nu_{0}, \mu\right)$ and the definition of barycentric projection are both utilized to obtain the right hand side of (21). We have also used the Lebesgue dominated convergence theorem and the fact that $\left\{\eta \circ \nabla \varphi_{s}\right\}$ is bounded in $L^{\infty}\left(\nu_{0}\right)$. 
Theorem 1 (Euler-Monge-Ampère system). If $\sigma$ minimizes $\mathcal{A}_{T}$ over $\mathcal{C}_{T}\left(\bar{\sigma}_{0}, \bar{\sigma}_{T}\right)$, then

$$
\partial_{t}\left(\sigma_{t} \mathbf{v}_{t}\right)+\nabla_{y} \cdot\left(\sigma_{t} \mathbf{v}_{t} \otimes \mathbf{v}_{t}\right)=\sigma_{t}\left[\bar{\gamma}^{t}-\mathbf{i d}\right]
$$

in the distributional sense, where $\gamma^{t} \in \Gamma_{o}\left(\nu_{0}, \sigma_{t}\right)$.

Proof. We use lemmas 8, 9 and the fact that $\sigma$ minimizes $\mathcal{A}_{T}$ to obtain

$$
\begin{aligned}
0 \leq \liminf _{s \rightarrow 0^{+}} \frac{\mathcal{A}_{T}\left(\sigma^{s}\right)-\mathcal{A}_{T}(\sigma)}{s} & \leq \int_{0}^{T} d t\left(\int_{\mathbb{R}^{d}}\left\langle\mathbf{v}_{t}, \partial_{t} \xi_{t}+\nabla_{y} \xi_{t} \mathbf{v}_{t}\right\rangle\right) d \sigma_{t} \\
& -\int_{0}^{T}\left(\int_{\mathbb{R}^{d}}\left\langle\mathbf{i d}-\bar{\gamma}^{t}, \xi_{t}\right\rangle\right) d \sigma_{t} d t .
\end{aligned}
$$

Since we can substitute $\xi$ by $-\xi$, the conclusion of the theorem follows.

\subsection{Another action as a quadratic form in $A C^{2}\left(0, T ; L^{2}\left(\nu_{0}\right)\right)$}

Throughout this subsection, $d=1$ and $\bar{\sigma}_{0}, \bar{\sigma}_{T} \in \mathcal{P}_{2}(\mathbb{R})$. We will not directly study minimizers of the action $\mathcal{A}_{T}$ over $\mathcal{C}_{T}\left(\bar{\sigma}_{0}, \bar{\sigma}_{T}\right)$ for two reasons. The first reason is that unlike the second order moment, the second term $\mu \rightarrow \int_{\mathbb{R}^{2}}|y-\bar{y}| d \mu(y) d \mu(\bar{y})$ appearing in the Wasserstein distance (5) is not differentiable. Note that, so far, we only know that (25) is a necessary condition satisfied by the minimizer of $\mathcal{A}_{T}$. To obtain an Euler-Lagrange equation which characterizes completely the minimizers of $\mathcal{A}_{T}$, we regularize $W_{2}$ to obtain a differentiable function

$$
\mathcal{W}^{s}(\mu):=\int_{\mathbb{R}}|y|^{2} d \mu(y)-\frac{1}{2 s} \int_{\mathbb{R}^{2}}|y-\bar{y}|^{s} d \mu(y) d \mu(\bar{y})+\frac{1}{12} .
$$

We introduce the corresponding action

$$
\mathcal{A}_{T}^{s}(\sigma):=\frac{1}{2} \int_{0}^{T}\left[\left|\sigma^{\prime}\right|^{2}(t)-\mathcal{W}^{s}\left(\sigma_{t}\right)\right] d t
$$

and later study its minimizers as $s$ tends to 1 . The second reason is that the term $-1 / 2 W_{2}^{2}\left(\nu_{0}, \cdot\right)$ appearing in $\mathcal{A}_{T}$ is not lower semicontinuous for the narrow convergence on $\mathcal{P}_{2}(\mathbb{R})$. This is a source of further difficulty we encounter while trying to directly minimize $\mathcal{A}_{T}$.

Let $M \in A C^{2}\left(0, T ; L^{2}\left(\nu_{0}\right)\right)$ be such that $M_{t}: X \rightarrow \mathbb{R}$ is the unique $\left(\nu_{0^{-}}\right.$ almost everywhere) monotone nondecreasing map satisfying $M_{t \#} \nu_{0}=\sigma_{t}$. We decompose $\mathcal{A}_{T}$ into two terms which will satisfy some lower semicontinuity properties in a sense to be made precise. These are

$$
\frac{1}{2}\left\|\sigma^{\prime}\right\|_{\text {metric }}^{2}-\frac{1}{2} \int_{0}^{T} \int_{X} y^{2} d \sigma_{t}(y) \text { and } \frac{1}{4} \int_{0}^{T} d t \int_{\mathbb{R}^{2}}|y-\bar{y}| d \sigma_{t}(y) d \sigma_{t}(\bar{y}) \text {. }
$$

The second term can be expressed as a function of $M$ :

$$
\mathcal{C}(M)=\frac{1}{4} \int_{X_{T}^{2}}\left|M_{t} x-M_{t} \bar{x}\right| d x d \bar{x} d t
$$


It is a convex functional on the Hilbert space $H^{1}\left(0, T, L^{2}\left(\nu_{0}\right)\right)$ with the standard inner product. Lemma 1 yields that the first expression is the quadratic form $\mathcal{Q}(M)=\mathcal{B}(M, M)$ where $\mathcal{B}$ is the bilinear form defined on $H^{1}\left(0, T, L^{2}\left(\nu_{0}\right)\right)$ by

$$
\mathcal{B}(M, N)=\frac{1}{2} \int_{0}^{T}\left(\left\langle M^{\prime}, N^{\prime}\right\rangle_{\nu_{0}}-\langle M, N\rangle_{\nu_{0}}\right) d t, \quad \mathcal{Q}(M)=\mathcal{B}(M, M) .
$$

We regularize $\mathcal{C}$ to obtain a differentiable convex functional

$$
\mathcal{C}^{s}(M)=\frac{1}{4 s} \int_{X_{T}^{2}}\left|M_{t} x-M_{t} \bar{x}\right|^{s} d t d x d \bar{x}
$$

We shall study the action $\mathcal{Q}(M)+\mathcal{C}^{s}(M)$ on $A C^{2}\left(0, T ; L^{2}\left(\nu_{0}\right)\right)$.

Remark 4. Let $\bar{M}_{0}, \bar{M}_{T} \in L^{2}\left(\nu_{0}\right)$ and let $M, \tilde{M} \in \mathcal{C}_{T}\left(\bar{M}_{0}, \bar{M}_{T}\right)$.

(a) If and $\alpha \in[0,1]$, then

$$
\mathcal{Q}((1-\alpha) M+\alpha \tilde{M})=\mathcal{Q}(M)+\alpha^{2} \mathcal{Q}(\tilde{M}-M)+2 \alpha \mathcal{B}(M, \tilde{M}-M) .
$$

We apply Poincare's inequality (with $s_{0}=0$ ) in proposition 2 to $\mathcal{S}=L^{2}\left(\nu_{0}\right)$ and obtain

$$
2 \mathcal{Q}(\tilde{M}-M) \geq\left(1-\frac{T^{2}}{\pi^{2}}\right)\left\|(\tilde{M}-M)^{\prime}\right\|_{m e t r i c}^{2}
$$

We combine (28) and (29) to obtain: if $M \neq \tilde{M}$, then

$$
\alpha \rightarrow \mathcal{Q}((1-\alpha) M+\alpha \tilde{M}) \quad \text { is strictly convex. }
$$

(b) In particular, if we set $M_{t}=(1-t / T) \bar{M}_{0}+(t / T) \bar{M}_{T}$, then $(28)(\alpha=1)$ and (29) imply

$$
\begin{aligned}
\mathcal{Q}(\tilde{M})-\mathcal{Q}(M) & -2 \mathcal{B}(M, \tilde{M}-M) \\
\geq & \frac{1}{2}\left(1-\frac{T^{2}}{\pi^{2}}\right)\left(\left\|\tilde{M}^{\prime}\right\|_{\text {metric }}^{2}-\frac{1}{T}\left\|\bar{M}_{T}-\bar{M}_{0}\right\|_{L^{2}\left(\nu_{0}\right)}^{2}\right) .
\end{aligned}
$$

We have used $\left\|(\tilde{M}-M)^{\prime}\right\|_{m e t r i c}^{2}=\left\|\tilde{M}^{\prime}\right\|_{m e t r i c}^{2}-(1 / T)\left\|\bar{M}_{T}-\bar{M}_{0}\right\|_{\nu_{0}}^{2}$.

Proposition 5 ( $\mathcal{Q}$ and $\mathcal{Q}+\mathcal{C}^{s}$ are sequentially weakly lower semicontinuous). Let $s \geq 1$ and let $\{M\} \cup\left\{M^{n}\right\}_{n=1}^{\infty} \subset \mathcal{C}_{T}\left(\bar{M}_{0}, \bar{M}_{T}\right)$. Suppose that for each $t \in(0, T),\left\{M_{t}^{n}\right\}_{n=1}^{\infty}$ converges weakly to $M_{t}$ in $L^{2}\left(\nu_{0}\right)$. Then,

$\liminf _{n \rightarrow+\infty} \mathcal{Q}\left(M^{n}\right) \geq \mathcal{Q}(M)$ and $\liminf _{n \rightarrow+\infty} \mathcal{Q}\left(M^{n}\right)+\mathcal{C}^{s}\left(M^{n}\right) \geq \mathcal{Q}(M)+\mathcal{C}^{s}(M)$. 
Proof. We assume without loss of generality that $\left\{\mathcal{Q}\left(M^{n}\right)\right\}_{n=1}^{\infty}$ is bounded independently of $n$. Poincaré's inequality (16) ensures that

$$
\sup _{n \in \mathbf{N}}\left\|\left(M^{n}\right)^{\prime}\right\|_{\text {metric }}, \quad \sup _{n \in \mathbf{N}}\left\|M^{n}\right\|_{L^{2}(\nu)}<\infty .
$$

Lemma 3 shows that the sequence $\left\{M^{n}\right\}_{n=1}^{\infty}$ converges weakly to $M$ in $L^{2}(\nu)$ and $\left\{\left(M^{n}\right)^{\prime}\right\}_{n=1}^{\infty}$ converges weakly to $M^{\prime}$ in $L^{2}(\nu)$. Note that, by (28),

$$
\mathcal{Q}\left(M^{n}\right) \geq \mathcal{Q}(M)+2 \mathcal{B}\left(M, M^{n}-M\right),
$$

where we have used (29) to ensure that $\mathcal{Q}\left(M^{n}-M\right) \geq 0$. Since $\mathcal{B}(M, \cdot)$ is linear and continuous on $H^{1}\left(0, T, L^{2}\left(\nu_{0}\right)\right)$, (32) gives that

$$
\liminf _{n \rightarrow+\infty} \mathcal{Q}\left(M^{n}\right) \geq \mathcal{Q}(M)
$$

Setting $E^{n}(x, y, t):=M_{t}^{n}(x)-M_{t}^{n}(y)$ we obtain that $\left\{E^{n}\right\}_{n=1}^{\infty}$ converges weakly to $E$ in $L^{2}\left(\nu \times \nu_{0}\right)$, where $E(x, y, t):=M_{t} x-M_{t} y$. This, together with the lower semicontinuity of the $L^{s}\left(\nu \times \nu_{0}\right)$-norm, yields

$$
\liminf _{n \rightarrow+\infty} \mathcal{C}^{s}\left(M^{n}\right) \geq \mathcal{C}^{s}(M)
$$

This concludes the proof of the proposition.

\section{Lagrangian minimizing paths in $\mathcal{P}_{2}(\mathbb{R})$}

Throughout this section, $d=1$ and so, $X=(-1 / 2,1 / 2), \nu_{0}=\left.\mathcal{L}^{1}\right|_{X}$ and $\nu=\left.\mathcal{L}^{2}\right|_{X_{T}}$. We suppose that $T \in(0, \pi)$. It is convenient to introduce $\mathcal{M}$ on, the set of monotone nondecreasing functions $M \in L^{2}\left(\nu_{0}\right)$.

\subsection{More on properties of paths in $A C^{2}\left(0, T ; \mathcal{P}_{2}(\mathbb{R})\right)$}

The method of proof for most of the results in this subsection exploits strongly that $d=1$. As far as we know, some of them, such as proposition 6 , are not available in the literature. The main point of that proposition is that (33) holds although the velocity $v$ may fail to be smooth in any sense. We use $\mathcal{Q}$ and $\mathcal{C}^{s}$ as defined in the previous section.

Suppose that $\bar{M}_{0}, \bar{M}_{T} \in \mathcal{M}$ on. The purpose of the next remark is to show that the minimizer of $\mathcal{Q}+\mathcal{C}^{s}$ over $\mathcal{C}\left(\bar{M}_{0}, \bar{M}_{T}\right)$ coincides with its minimizer over $\mathcal{C}\left(\bar{M}_{0}, \bar{M}_{T}\right) \cap\left\{M \mid M_{t} \in \mathcal{M o n}\right\}$.

Remark 5. Suppose $\tilde{M} \in A C^{2}\left(0, T ; L^{2}\left(\nu_{0}\right)\right)$ and set $\sigma_{t}=\tilde{M}_{t \#} \nu_{0}$ so that by remark $1, \sigma \in A C^{2}\left(0, T ; \mathcal{P}_{2}(\mathbb{R})\right)$. Let $M_{t} \in \mathcal{M}$ on be such that $M_{t \#} \nu_{0}=\sigma_{t}$. By lemma $1, M \in A C^{2}\left(0, T ; L^{2}\left(\nu_{0}\right)\right)$. We combine remark 1 and lemma 1 to conclude that $\left\|M^{\prime}\right\|_{\text {metric }} \leq\left\|\tilde{M}^{\prime}\right\|_{\text {metric }}$. Since

$$
\left\|M_{t}\right\|_{L^{2}\left(\nu_{0}\right)}=\int_{\mathbb{R}}|z|^{2} d \sigma_{t}(z)=\left\|\tilde{M}_{t}\right\|_{L^{2}\left(\nu_{0}\right)},
$$


it is apparent that $\mathcal{Q}(M) \leq \mathcal{Q}(\tilde{M})$. Similarly,

$$
\mathcal{C}^{s}(M)=\frac{1}{4 s} \int_{0}^{T} \int_{\mathbb{R}^{2}}|z-w|^{s} d \sigma_{t}(z) d \sigma_{t}(w)=\mathcal{C}^{s}(\tilde{M})
$$

and so, $\mathcal{Q}(M)+\mathcal{C}^{s}(M) \leq \mathcal{Q}(\tilde{M})+\mathcal{C}^{s}(\tilde{M})$. We use lemma 1 to obtain that $\mathcal{A}_{T}^{s}(\sigma)=\mathcal{Q}(M)+\mathcal{C}^{s}(M)-T / 24$.

Proposition 6. Suppose $\sigma \in A C^{2}\left(0, T ; \mathcal{P}_{2}(\mathbb{R})\right)$. Let $v$ be the Borel velocity of minimal norm associated to $\sigma$ and let $M_{t} \in \mathcal{M}$ on be such that $M_{t \#} \nu_{0}=$ $\sigma_{t}$. For each $t$, modifying $M_{t}$ on a countable subset of $X$ if necessary, we may assume without loss of generality that $M_{t}$ is left continuous (see subsection 2.2). Then, we have

$$
M_{t}^{\prime} x=v_{t}\left(M_{t} x\right)
$$

for $\nu$-almost every $(x, t) \in X_{T}$.

Proof. First, recall that $M \in A C^{2}\left(0, T ; L^{2}\left(\nu_{0}\right)\right)$. Let $A$ and the sequence $\left\{h_{k}^{+}\right\}$be given as in lemma 6 and let $\mathcal{K}$ be a countable dense subset of $C_{c}^{1}(\mathbb{R})$. We use the definition of $v$ and lemma 6 to obtain a Borel set $J \subset(0, T)$ of full measure satisfying the following for each $t \in J: \frac{d}{d t} \int_{\mathbb{R}} \varphi d \sigma_{t}=\int_{\mathbb{R}} v_{t} \varphi^{\prime} d \sigma_{t}$ for all $\varphi \in \mathcal{K}$, and the set

$$
D_{t}:=\left\{x \in X:(x, t) \in A \text { and } x \text { is a Lebesgue point of } M_{t}^{\prime}\right\} \subset X
$$

has full $\nu_{0}$-measure. For $t \in J$, let us define

$$
\bar{M}_{t} x=\liminf _{n \rightarrow \infty}\left(n \int_{x-\frac{1}{n}}^{x} M_{t}^{\prime} \bar{x} d \bar{x}\right), \quad x \in X .
$$

Note that $\bar{M}_{t}$ is a Borel map as a liminf of continuous functions on $X$. We also define $S_{t}(z)=-1 / 2+\sigma_{t}(-\infty, z]$, which is, up to an additive constant, the right continuous distribution function of $\sigma_{t}$. Then let us introduce the map $w_{t}=\bar{M}_{t} \circ S_{t}$, which is a Borel map as a composition of two Borel maps. Fix $t \in J$ arbitrary. For $x \in X$, we have

$$
w_{t}\left(M_{t} x\right)=\bar{M}_{t} \circ S_{t} \circ M_{t} x=\bar{M}_{t} x_{t}^{r},
$$

where $x_{t}^{r}$ is the right endpoint of $I_{t, x}:=\left\{\bar{x} \in X: M_{t} \bar{x}=M_{t} x\right\}$. Notice that since $M_{t}$ is left continuous, $I_{t, x}$ is a right closed interval (possibly degenerate) containing $x$. Now assume that $x \in D_{t}$. Then if $I_{t, x}$ contains only $x=x_{t}^{r}$, we obtain $w_{t}\left(M_{t} x\right)=M_{t}^{\prime} x$ as a direct consequence of (34). Otherwise $I_{t, x}$ is an interval of positive length so that $x_{t}^{r}$ is the largest value in the interval. Thus, $\left[x_{t}^{r}-1 / n, x_{t}^{r}\right] \subset I_{t, x}$ for $n$ large enough. Because $D_{t}$ has full $\nu_{0}$-measure, lemma 7 gives that $\int_{x_{t}^{r}-\frac{1}{n}}^{x_{r}^{r}} M_{t}^{\prime} \bar{x} d \bar{x}=(1 / n) M_{t}^{\prime} x$ and so, $\bar{M}_{t} x_{t}^{r}=M_{t}^{\prime} x$. This together with (34) yields

$$
w_{t}\left(M_{t} x\right)=M_{t}^{\prime} x \text { for all } x \in D_{t} .
$$


We combine (14) and (35) to obtain for $t \in J$ and $\varphi \in \mathcal{K}$,

$$
\begin{aligned}
\int_{\mathbb{R}} w_{t} \varphi^{\prime} d \sigma_{t} & =\int_{X} w_{t}\left(M_{t} x\right) \varphi^{\prime}\left(M_{t} x\right) d x=\int_{X} M_{t}^{\prime} x \varphi^{\prime}\left(M_{t} x\right) d x \\
& =\lim _{k \rightarrow \infty} \int_{X} \frac{\varphi\left(M_{t+h_{k}^{+}} x\right)-\varphi\left(M_{t} x\right)}{h_{k}^{+}} d x \\
& =\lim _{k \rightarrow \infty} \int_{\mathbb{R}} \varphi \frac{d \sigma_{t+h_{k}^{+}}-d \sigma_{t}}{h_{k}^{+}}=\int_{\mathbb{R}} v_{t} \varphi^{\prime} d \sigma_{t} .
\end{aligned}
$$

As $\mathcal{K}$ is dense in $C_{c}^{1}(\mathbb{R})$, we conclude that the equalities in (36) hold for all $\varphi \in C_{c}^{1}(\mathbb{R})$. As $\sigma_{t} \in \mathcal{P}_{2}(\mathbb{R})$ is a Borel probability measure on $\mathbb{R},\left\{\varphi^{\prime} \mid \varphi \in\right.$ $\left.C_{c}^{1}(\mathbb{R})\right\}$ is itself dense in $L^{2}\left(\sigma_{t}\right)$. Here, we have used that $\mathbb{R}$ is of dimension 1. Hence (36) holds if we substitute $\varphi^{\prime}$ by any element of $L^{2}\left(\sigma_{t}\right)$. This proves that $w_{t}=v_{t} \sigma_{t}$-almost everywhere. Moreover, observe that if $\sigma_{t}(\mathcal{N})=0$ then $\nu_{0}\left(M_{t}^{-1}(\mathcal{N})\right)=\sigma_{t}(\mathcal{N})=0$. Therefore, for $t \in J$, using (35) we conclude that $M_{t}^{\prime} x=w_{t}\left(M_{t} x\right)=v_{t}\left(M_{t} x\right)$ for $\nu_{0}$-almost every $x \in X$. This yields the proposition.

\subsection{Minimizing paths on $\mathcal{P}_{2}(\mathbb{R})$}

We recall that $\mathcal{Q}$ and $\mathcal{C}^{s}$ are defined in section 3.2. Since $T<\pi$, it follows from the Poincaré inequality that sublevel subsets of $\mathcal{Q}+\mathcal{C}^{s}$ are contained in sublevel subsets of $\|\cdot\|_{A C^{2}\left(0, T ; L^{2}\left(\nu_{0}\right)\right)}$. Observe that $\mathcal{Q}+\mathcal{C}^{s}$ is differentiable only for $s>1$. We will see that if $\bar{M}_{0}, \bar{M}_{T}$ are monotone nondecreasing satisfying $M_{0 \#} \nu_{0}=\bar{\sigma}_{0}$ and $M_{T \#} \nu_{0}=\bar{\sigma}_{T}$, then minimizing $\mathcal{A}_{T}$ over $\mathcal{C}_{T}\left(\bar{\sigma}_{0}, \bar{\sigma}_{T}\right)$ is equivalent to minimizing $\mathcal{Q}+\mathcal{C}$ over $\mathcal{C}_{T}\left(\bar{M}_{0}, \bar{M}_{T}\right)$. Furthermore, there is a unique minimizer in each one of these two problems. We analyze the minimizers of $\mathcal{Q}+\mathcal{C}$ by first studying those of $\mathcal{Q}+\mathcal{C}^{s}$.

Theorem 2. Suppose $\bar{M}_{0}, \bar{M}_{T} \in L^{2}\left(\nu_{0}\right)$. Then $\mathcal{Q}+\mathcal{C}^{s}$ admits a unique minimizer $M^{s}$ on $\mathcal{C}_{T}\left(\bar{M}_{0}, \bar{M}_{T}\right)$. If we further assume that $\bar{M}_{0}, \bar{M}_{T} \in \mathcal{M}$ on then for $\mathcal{L}^{1}$-almost every $t \in(0, T), M_{t}^{s} \in \mathcal{M}$ on.

Proof. We choose $\mathcal{S}=L^{2}\left(\nu_{0}\right)$ with the metric dist $=\|\cdot\|_{\nu_{0}}$. We take $\tau$ to be the weak topology and we apply proposition 3 . Set $M_{t}=(1-$ $t / T) \bar{M}_{0}+(t / T) \bar{M}_{T}$ and let $\left\{M^{n}\right\}_{n=1}^{\infty}$ be a minimizing sequence for $\mathcal{Q}+\mathcal{C}^{s}$ over $\mathcal{C}_{T}\left(\bar{M}_{0}, \bar{M}_{T}\right)$. We may assume that $1+\mathcal{Q}(M)+\mathcal{C}^{s}(M) \geq \mathcal{Q}\left(M^{n}\right)+$ $\mathcal{C}^{s}\left(M^{n}\right)$. It follows from the Poincare's inequalities (15)-(16) that $\left\{M^{n}\right\}_{n=1}^{\infty}$ is bounded in $H^{1}\left(0, T ; L^{2}\left(\nu_{0}\right)\right)$. In particular, there exists a constant $C$ independent of $n$ such that

$$
\left\|\left|\left(M^{n}-M\right)^{\prime}\right|\right\|_{L^{2}(0, T)} \leq C .
$$

Thanks to (37) we can apply proposition 4 . We obtain a subsequence of $\left\{M^{n}\right\}_{n=1}^{\infty}$ (we do not relabel) such that for each $t \in[0, T],\left\{M_{t}^{n}\right\}_{n=1}^{\infty}$ converges weakly to some $M_{t}^{s}$ in $L^{2}\left(\nu_{0}\right)$. We use proposition 3 to obtain that $M^{s} \in A C^{2}\left(0, T ; L^{2}\left(\nu_{0}\right)\right)$. One can readily conclude that $M^{s} \in \mathcal{C}_{T}\left(\bar{M}_{0}, \bar{M}_{T}\right)$. 
By lemma 3, $\left\{M^{n}\right\}_{n=1}^{\infty}$ converges weakly to $M^{s}$ in $L^{2}(\nu)$ and $\left\{\left(M^{n}\right)^{\prime}\right\}_{n=1}^{\infty}$ converges weakly to $\left(M^{s}\right)^{\prime}$ in $L^{2}\left(\nu_{0}\right)$. By proposition $5, \mathcal{Q}+\mathcal{C}^{s}$ is sequentially weakly lower semicontinuous on $\mathcal{C}_{T}\left(\bar{M}_{0}, \bar{M}_{T}\right)$. We use these to conclude that $M^{s}$ minimizes $\mathcal{Q}+\mathcal{C}^{s}$ over $\mathcal{C}_{T}\left(\bar{M}_{0}, \bar{M}_{T}\right)$. By remark $4, \mathcal{Q}$ is strictly convex on $\mathcal{C}_{T}\left(\bar{M}_{0}, \bar{M}_{T}\right)$. Hence $\mathcal{Q}+\mathcal{C}^{s}$ is strictly convex as the sum of a strictly convex function and a convex function. This proves that the minimizer $M^{s}$ is unique.

Suppose, in addition, that $\bar{M}_{0}, \bar{M}_{T} \in \mathcal{M}$ on. Let $\tilde{M}^{s}$ be such that $\tilde{M}_{t}^{s}$ is monotone nondecreasing and $\tilde{M}_{t \#}^{s} \nu_{0}=M_{t \#}^{s} \nu_{0}$. By remark $5, \tilde{M} \in$ $A C^{2}\left(0, T ; L^{2}\left(\nu_{0}\right)\right)$ and $\mathcal{Q}(\tilde{M})+\mathcal{C}^{s}(\tilde{M}) \leq \mathcal{Q}(M)+\mathcal{C}^{s}(M)$. Thus $\tilde{M}$ is another minimizer of $\mathcal{Q}+\mathcal{C}^{s}$ over $\mathcal{C}_{T}\left(\bar{M}_{0}, \bar{M}_{T}\right)$. By uniqueness, $M^{s}=\tilde{M}^{s}$. This proves that for $\mathcal{L}^{1}$-almost every $t \in(0, T), M_{t}^{s}$ is monotone nondecreasing.

Remark 6. Suppose $M \in A C^{2}\left(0, T ; L^{2}\left(\nu_{0}\right)\right)$ so that $M \in L^{2}(\nu)$. If, in addition, $M_{t} \in \mathcal{M}$ on for each $t \in[0, T]$ then that for $0<r<1 / 2$, we have $r\left|M_{t} x\right| \leq \int_{X}\left|M_{t} y\right| d y$ on $[-1 / 2+r, 1 / 2-r]=: X^{r}$. Hence,

$$
r \int_{-1 / 2+r}^{1 / 2-r}\left|\partial_{x} M_{t} x\right| \leq r\left(M_{t}(1 / 2-r)-M_{t}(-1 / 2+r)\right) \leq 2 \int_{X}\left|M_{t} y\right| d y .
$$

We have used that $\partial_{x} M_{t}$ is a nonnegative measure on $X$. Thus, $r\left\|\partial_{x} M\right\|_{X_{T}^{r}} \leq$ $2 \sqrt{T}\|M\|_{\nu}$. Let $\partial_{t} M$ be the distributional derivative of $M$ which coincides with $M^{\prime}$ by remark 2 . Then $\left\|\partial_{t} M\right\|_{\nu}=\left\|M^{\prime}\right\|_{\text {metric }}$. This proves the existence of a constant $\bar{C}(r)$ dependent on $T$ but independent of $M$ such that

$$
\|M\|_{B V\left(X_{T}^{r}\right)} \leq \bar{C}(r)\|M\|_{A C^{2}\left(0, T ; L^{2}\left(\nu_{0}\right)\right)} .
$$

\subsection{Euler-Poisson system in 1-d in terms of its associated flow}

Suppose $s>1$, so that $\mathcal{Q}+\mathcal{C}^{s}$ is not only strictly convex, but also Gâteaux differentiable. If $\bar{M}_{0}, \bar{M}_{T} \in L^{2}\left(\nu_{0}\right)$, standard arguments give that $M^{s} \in \mathcal{C}_{T}\left(\bar{M}_{0}, \bar{M}_{T}\right)$ minimizes $\mathcal{Q}+\mathcal{C}^{s}$ over $\mathcal{C}_{T}\left(\bar{M}_{0}, \bar{M}_{T}\right)$ if and only if

$$
\left(M^{s}\right)_{t}^{\prime \prime} x+M_{t}^{s} x=\frac{1}{2} \int_{X}\left(M_{t}^{s} x-M_{t}^{s} y\right)\left|M_{t}^{s} x-M_{t}^{s} y\right|^{s-2} d y
$$

in the sense of distributions on $X_{T}$. An analogous characterization of $M^{1}$ can be stated although $\mathcal{Q}+\mathcal{C}^{1}$ is not Gâteaux differentiable everywhere. As in the previous section, let us set $X^{r}=[-1 / 2+r, 1 / 2-r]$.

Proposition 7. Suppose $M \in \mathcal{C}_{T}\left(\bar{M}_{0}, \bar{M}_{T}\right), W$ is a Borel function on $X_{T}^{2}$ such that $W(x, y, t) \in \partial .|\cdot|\left(M_{t} x-M_{t} y\right)$ and $W(x, y, t)=-W(y, x, t)$ for $\nu \times \nu_{0}$ - almost every $(x, y, t) \in X_{T}^{2}$. If

$$
M_{t}^{\prime \prime} x+M_{t} x=\frac{1}{2} \int_{X} W(x, \bar{x}, t) d \bar{x}
$$

in the sense of distributions on $X_{T}$, then $M$ is the unique minimizer of $\mathcal{Q}+\mathcal{C}$ over $\mathcal{C}_{T}\left(\bar{M}_{0}, \bar{M}_{T}\right)$. If we further assume that $\bar{M}_{0}, \bar{M}_{T}$ are monotone nondecreasing, then for $\mathcal{L}^{1}$-almost every $t \in(0, T), M_{t}$ is monotone nondecreasing. 
Proof. Let $\tilde{M} \in \mathcal{C}_{T}\left(\bar{M}_{0}, \bar{M}_{T}\right)$ be arbitrary. Since $W(x, y, t)=-W(y, x, t)$, we have

$$
\begin{aligned}
\int_{X_{T}^{2}} W(x, \bar{x}, t)\left(\tilde{M}_{t} x\right. & \left.-M_{t} x\right) d x d \bar{x} d t \\
& =-\int_{X_{T}^{2}} W(x, \bar{x}, t)\left(\tilde{M}_{t} \bar{x}-M_{t} \bar{x}\right) d x d \bar{x} d t .
\end{aligned}
$$

By the definition of $\mathcal{B}$ in (27) and (41),

$$
-8 \overline{\mathcal{B}}(M, \tilde{M}-M)=\int_{X_{T}^{2}} W(x, \bar{x}, t)\left(\left(\tilde{M}_{t} x-\tilde{M}_{t} \bar{x}\right)-\left(M_{t} x-M_{t} \bar{x}\right)\right) d x d \bar{x} d t .
$$

We exploit (42) and the fact that $W(x, y, t) \in \partial .|\cdot|\left(M_{t} x-M_{t} y\right)$ to conclude that

$$
\frac{1}{4} \int_{X_{T}^{2}}\left(\left|\tilde{M}_{t} x-\tilde{M}_{t} \bar{x}\right|-\left|M_{t} x-M_{t} \bar{x}\right|\right) d x d \bar{x} d t \geq-2 \mathcal{B}(M, \tilde{M}-M) .
$$

This, in view of (28) (set $\alpha=1)$ and the fact that $\mathcal{Q}(\tilde{M}-M) \geq 0$, yields $\mathcal{Q}(\tilde{M})+\mathcal{C}(\tilde{M}) \geq \mathcal{Q}(M)+\mathcal{C}(M)$. Hence, $M$ minimizes $\mathcal{Q}+\mathcal{C}$ over $\mathcal{C}_{T}\left(\bar{M}_{0}, \bar{M}_{T}\right)$. For each $t \in[0, T]$, define $M_{t}^{o}$ to be the unique monotone nondecreasing map such that $M_{t \#}^{o} \nu_{0}=M_{t \#} \nu_{0}=: \sigma_{t}$. By remark 1, the fact that $M \in A C^{2}\left(0, T ; L^{2}\left(\nu_{0}\right)\right)$ yields $\sigma \in A C^{2}\left(0, T ; \mathcal{P}_{2}(\mathbb{R})\right)$. Lemma 1 ensures that $M^{o} \in \mathcal{C}_{T}\left(\bar{M}_{0}, \bar{M}_{T}\right)$ and

$$
\left\|M^{\prime}\right\|_{\text {metric }} \geq\left\|\sigma^{\prime}\right\|_{\text {metric }}=\left\|\left(M^{o}\right)^{\prime}\right\|_{\text {metric }} .
$$

This, along with the fact $\mathcal{C}(M)=\mathcal{C}\left(M^{o}\right)$, yields that $M^{o}$ is also a minimizer of $\mathcal{Q}+\mathcal{C}$ over $\mathcal{C}_{T}\left(\bar{M}_{0}, \bar{M}_{T}\right)$. The strict convexity of $\mathcal{Q}+\mathcal{C}$ obtained in (30) ensures uniqueness of its minimizer over $\mathcal{C}_{T}\left(\bar{M}_{0}, \bar{M}_{T}\right)$. Thus, $M=M^{o}$.

We are next going to show the converse of proposition 7 . For that, let $\bar{M}=(1-t / T) \bar{M}_{0}+(t / T) \bar{M}_{T}$. In this work we are interested in what happens when $s \downarrow 1$; we shall assume that $s \leq 2$. Observe that for $s \in[1,2]$ we have

$$
\sup _{s^{\prime} \in[1,2]} \mathcal{Q}(\bar{M})+\mathcal{C}^{s^{\prime}}(\bar{M}) \geq \mathcal{Q}\left(M^{s}\right)+\mathcal{C}^{s}\left(M^{s}\right) \geq \mathcal{Q}\left(M^{s}\right),
$$

where $M^{s}$ is the minimizer of $\mathcal{Q}+\mathcal{C}^{s}$ over $\mathcal{C}_{T}\left(\bar{M}_{0}, \bar{M}_{T}\right)$. Hence Poincare's inequality (16) yields

$$
\sup _{s \in[1,2]}\left(\left\|\left(M^{s}\right)^{\prime}\right\|_{m e t r i c}+\sup _{t \in[0, T]}\left\|M_{t}^{s}\right\|_{\nu_{0}}^{2}\right)<\infty .
$$

The above supremum depends only on $T, \bar{M}_{0}$ and $\bar{M}_{T}$. 
Theorem 3. Suppose $\bar{M}_{0}, \bar{M}_{T} \in L^{2}\left(\nu_{0}\right) \cap \mathcal{M}$ on and $M^{1} \in \mathcal{C}_{T}\left(\bar{M}_{0}, \bar{M}_{T}\right)$. Then $M^{1}$ minimizes $\mathcal{Q}+\mathcal{C}$ over $\mathcal{C}_{T}\left(\bar{M}_{0}, \bar{M}_{T}\right)$ if and only if

$$
\left(M^{1}\right)^{\prime} \in A C^{2}\left(0, T ; L^{2}\left(\nu_{0}\right)\right)
$$

and

$$
\left(M^{1}\right)_{t}^{\prime \prime} x+M_{t}^{1} x=\int_{X} W_{M^{1}}(x, \bar{x}, t) d \bar{x}
$$

in the sense of distributions on $X_{T}$, for some $W_{M^{1}} \in L^{\infty}\left(X_{T}^{2}\right)$ satisfying $W_{M^{1}}(x, \bar{x}, t) \in \partial .|\cdot|\left(M_{t}^{1} x-M_{t}^{1} \bar{x}\right)$ and $W_{M^{1}}(x, \bar{x}, t)=-W_{M^{1}}(\bar{x}, x, t)$ for $\nu \times \nu_{0}$ - almost every $(x, \bar{x}, t) \in X_{T}^{2}$.

Proof. Let $M^{s}$ be the minimizer of $\mathcal{Q}+\mathcal{C}^{s}$ over $\mathcal{C}_{T}\left(\bar{M}_{0}, \bar{M}_{T}\right)$. By the previous proposition, $M_{t}^{s} \in \mathcal{M}$ on. We set $X_{T}^{r}=(0, T) \times[-1 / 2+r, 1 / 2-r]$. We use (16) to obtain existence of a constant $\bar{C}(r)$ which depends on $r$ but is independent of $s \in[1,2]$ such that $\left\|M^{s}\right\|_{B V\left(X_{T}^{r}\right)} \leq \bar{C}(r)$. We next invoke the compactness of bounded subsets of $B V\left(X_{T}^{r}\right)$ in $L^{1}\left(X_{T}^{r}\right)$. We obtain existence of a sequence $\left\{s_{j}\right\}_{j=1}^{\infty} \subset[1,2]$ converging to 1 as $j$ tends to $+\infty$ and such that $\left\{M^{s_{j}}\right\}_{j=1}^{\infty}$ converges to some $\tilde{M}^{1}$ in $L_{l o c}^{1}\left(X_{T}\right)$. Passing to a subsequence if necessary, we may assume that $\left\{M^{s_{j}}\right\}_{j=1}^{\infty}$ converges $\nu$-almost everywhere to $\tilde{M}^{1}$. Passing to another subsequence, it is easy to check that $\tilde{M}^{1} \in \mathcal{C}_{T}\left(\bar{M}_{0}, \bar{M}_{T}\right)$ and $\left\{M_{t}^{s_{j}}\right\}_{j=1}^{\infty}$ converges weakly to $\tilde{M}_{t}^{1}$ in $L^{2}\left(\nu_{0}\right)$ for each $t \in(0, T)$. By Egoroff's theorem, $\left\{M^{s_{j}}\right\}_{j=1}^{\infty}$ converges uniformly to $\tilde{M}^{1}$ except on a set of $\nu$-small measure. By (43) $\left\{\left|M_{t}^{s_{j}} x-M_{t}^{s_{j}} y\right|^{s_{j}}\right\}_{j=1}^{\infty}$ is weakly closed in $L^{1}\left(\nu \times \nu_{0}\right)$ and so,

$$
\lim _{j \rightarrow+\infty} \int_{X_{T}^{2}}\left|M_{t}^{s_{j}} x-M_{t}^{s_{j}} \bar{x}\right|^{s_{j}} d t d x d \bar{x}=\int_{X_{T}^{2}}\left|\tilde{M}_{t}^{1} x-\tilde{M}_{t}^{1} \bar{x}\right| d t d x d \bar{x} .
$$

This, together with the fact that proposition 5 provides the lower semicontinuity of $\mathcal{Q}$, yields $\liminf \operatorname{in}_{j \rightarrow+\infty} \mathcal{Q}\left(M^{s_{j}}\right)+\mathcal{C}^{s_{j}}\left(M^{s_{j}}\right) \geq \mathcal{Q}\left(\tilde{M}^{1}\right)+\mathcal{C}\left(\tilde{M}^{1}\right)$. Hence, $\tilde{M}^{1}$ minimizes $\mathcal{Q}+\mathcal{C}$ over $\mathcal{C}_{T}\left(\bar{M}_{0}, \bar{M}_{T}\right)$. Uniqueness of minimizers being ensured by theorem 2 , we conclude that $\tilde{M}^{1}=M^{1}$. We use that

$\liminf _{j \rightarrow+\infty} \mathcal{Q}\left(M^{1}\right)+\mathcal{C}^{s_{j}}\left(M^{1}\right) \geq \liminf _{j \rightarrow+\infty} \mathcal{Q}\left(M^{s_{j}}\right)+\mathcal{C}^{s_{j}}\left(M^{s_{j}}\right) \geq \mathcal{Q}\left(M^{1}\right)+\mathcal{C}\left(M^{1}\right)$

and (45) to conclude that $\mathcal{Q}\left(M^{1}\right)=\liminf _{j \rightarrow+\infty} \mathcal{Q}\left(M^{s_{j}}\right)$. Using (29) we obtain

$$
0=\liminf _{j \rightarrow+\infty} \mathcal{Q}\left(M^{s_{j}}-M^{1}\right) \geq \liminf _{j \rightarrow+\infty} 1 / 2\left(1-\frac{T^{2}}{\pi^{2}}\right)\left\|\left(M^{s_{j}}-M^{1}\right)^{\prime}\right\|_{m e t r i c} .
$$

This, together with Poincaré's inequality (16) proves that $\left\{M^{s_{j}}\right\}_{j=1}^{\infty}$ converges strongly to $M^{1}$ in $H^{1}\left(0, T ; L^{2}\left(\nu_{0}\right)\right)$. Set

$$
W_{M^{s}}(x, \bar{x}, t):=\left(M_{t}^{s} x-M_{t}^{s} \bar{x}\right)\left|M_{t}^{s} x-M_{t}^{s} \bar{x}\right|^{s-2} .
$$


We have that $W_{M^{s}}$ is uniformly bounded in $L^{2}\left(X_{T}^{2}\right)$ and

$$
W_{M^{s}}(x, \bar{x}, t) \in \partial . G^{s}\left(M_{t}^{s} x-M_{t}^{s} \bar{x}\right) \text { with } G^{s}(e):=\frac{|e|^{s}}{s} .
$$

Hence, up to a subsequence we do not relabel, $\left\{W_{M^{s_{j}}}\right\}_{j=1}^{\infty}$ converges weakly in $L^{2}\left(X_{T}^{2}\right)$ to some $W_{M^{1}}$ in $L^{2}\left(X_{T}^{2}\right)$. The fact that $\left\{M^{s_{j}}\right\}_{j=1}^{\infty}$ converges strongly to $M^{1}$ in $H^{1}\left(0, T ; L^{2}\left(\nu_{0}\right)\right)$ yields $W_{M^{1}}(x, \bar{x}, t) \in \partial .|\cdot|\left(M_{t}^{1} x-M_{t}^{1} \bar{x}\right)$. Using (40) we obtain (44). It is apparent that $W_{M^{1}}(x, \bar{x}, t)=-W_{M^{1}}(\bar{x}, x, t)$ for $\nu \times \nu_{0}$-almost every $(x, \bar{x}, t) \in X_{T}^{2}$. We use proposition 7 to conclude that any solution of $(44)$ is a minimizer of $\mathcal{Q}+\mathcal{C}$. Observe that $W_{M^{1}} \in L^{\infty}\left(X_{T}^{2}\right)$ and

$$
\left|\int_{X} W_{M^{1}}(x, \bar{x}, t) d \bar{x}\right| \leq 1 \text {. }
$$

Since $M^{1} \in A C^{2}\left(0, T ; L^{2}\left(\nu_{0}\right)\right)$, (44) implies $\left(M^{1}\right)^{\prime} \in A C^{2}\left(0, T ; L^{2}\left(\nu_{0}\right)\right)$.

Remark 7. Let $M^{1}$ be as in theorem 3. Note that, in particular, if for $\mathcal{L}^{2}$ almost every $(x, t), M_{t}^{1} x \neq M_{t}^{1} \bar{x}$ for $\mathcal{L}^{1}$-almost every $\bar{x} \in X$, (44) reads off

$$
\left(M^{1}\right)_{t}^{\prime \prime} x+M_{t}^{1} x=x
$$

Corollary 1. Suppose $\bar{\sigma}_{0}, \bar{\sigma}_{T} \in \mathcal{P}_{2}(\mathbb{R})$. Let $\bar{M}_{0}, \bar{M}_{T}$ be monotone nondecreasing maps satisfying $\bar{M}_{0 \#} \nu_{0}=\bar{\sigma}_{0}$ and $\bar{M}_{T \#} \nu_{0}=\bar{\sigma}_{T}$, and let $M^{s}$ be the unique minimizer of $\mathcal{Q}+\mathcal{C}^{s}$ over $\mathcal{C}_{T}\left(\bar{M}_{0}, \bar{M}_{T}\right)$ given by theorem 2. Then $\sigma^{s}:=M_{t \#}^{s} \nu_{0}$ is the unique minimizer of $\mathcal{A}_{T}^{s}$ over $\mathcal{C}_{T}\left(\bar{\sigma}_{0}, \bar{\sigma}_{T}\right)$.

Proof. Let $\sigma \in A C^{2}\left(0, T ; \mathcal{P}_{2}(\mathbb{R})\right)$ be arbitrary and let $M_{t} \in \mathcal{M}$ on such that $M_{t \#} \nu_{0}=\sigma_{t}$. By remark $5, M \in A C^{2}\left(0, T ; L^{2}\left(\nu_{0}\right)\right)$. We have

$$
\mathcal{A}_{T}^{s}\left(\sigma^{s}\right)+T / 24=\mathcal{Q}\left(M^{s}\right)+\mathcal{C}^{s}\left(M^{s}\right) \leq \mathcal{Q}(M)+\mathcal{C}^{s}(M)=\mathcal{A}_{T}^{s}(\sigma)+T / 24 .
$$

The inequality in (46) being strict unless $M^{s}=M$, we conclude that $\sigma^{s}$ is the unique minimizer of $\mathcal{A}_{T}^{s}$ over $\mathcal{C}_{T}\left(\bar{\sigma}_{0}, \bar{\sigma}_{T}\right)$.

Suppose $\bar{\sigma}_{0}, \bar{\sigma}_{T} \in \mathcal{P}_{2}(\mathbb{R})$ and $\sigma^{1}$ is the unique minimizer of $\mathcal{A}_{T}$ found in corollary 1 . Let $v$ be the Borel velocity of minimal norm associated to $\sigma^{1}$. For each $t \in[0, T]$, let $M_{t}^{1}$ be the monotone nondecreasing map such that $M_{t \#}^{1} \nu_{0}=\sigma_{t}^{1}$. Since the tangent space $\mathcal{T}_{\sigma_{t}^{1}} \mathcal{P}_{2}(\mathbb{R})$ is a separable Hilbert space, we shall identify it with its dual and so, we can view the tangent vector $v_{t}$ as a cotangent vector. Although the Hamiltonian $H$ is defined on the cotangent bundle of $\mathcal{P}_{2}(\mathbb{R})$, now it still makes sense to write $H\left(\sigma_{t}^{1}, v_{t}\right)$. By remark 3 and lemma 1 , for $\mathcal{L}^{1}$-almost every $t \in(0, T)$

$$
2 H\left(\sigma_{t}^{1}, v_{t}\right)=\left\|v_{t}\right\|_{L^{2}(0, T)}^{2}+W_{2}^{2}\left(\sigma_{t}^{1}, \nu_{0}\right)=\int_{X}\left[\left|\left(M_{t}^{1}\right)^{\prime}\right|^{2}+\left|M_{t}^{1}-\mathbf{i d}\right|^{2}\right] d \nu_{0}
$$

Whereas $t \rightarrow H\left(\sigma_{t}^{1}, v_{t}\right)$ may not be continuous, the $H^{2}$ regularity of $M^{1}$ proved in theorem 3 ensures that the map

$$
t \rightarrow 2 \bar{H}(t):=\int_{X}\left[\left|\left(M^{1}\right)_{t}^{\prime}\right|^{2} d \nu_{0}+W_{2}^{2}\left(\sigma_{t}^{1}, \nu_{0}\right)\right] d \nu_{0}
$$


belongs to $H^{1}(0, T)$. As a matter of fact, we will prove that this map is a constant, by showing that its distributional derivative is null. We are able to show conservation of the Hamiltonian even without the assumption that $\sigma_{t}^{1}<<\mathcal{L}^{1}$, that in general fails. The proof relies on (33) of proposition 6 and on the following lemma.

Lemma 10. Suppose that $M: X \rightarrow \mathbb{R}$ is monotone nondecreasing and $W \in L^{\infty}\left(X_{T}^{2}\right)$ satisfy $W(x, \bar{x}) \in \partial .|\cdot|(M x-M \bar{x})$ and $W(x, \bar{x})=-W(\bar{x}, x)$ for $\mathcal{L}^{2}$-almost every $(x, \bar{x}) \in X^{2}$. If $\xi \in L^{1}(\sigma)$ is a Borel map for $\sigma:=M_{\#} \nu_{0}$, then

$$
2 \int_{X} x \xi(M x) d x=\int_{X^{2}} \xi(M x) W(x, \bar{x}) d x d \bar{x} .
$$

Proof. We set

$$
A=\{(x, \bar{x}) \mid M x>M \bar{x}\}, \quad B=\{(x, \bar{x}) \mid M x<M \bar{x}\} .
$$

We use that $M$ is monotone nondecreasing to conclude that

$$
\begin{aligned}
2 \int_{X} x \xi(M x) d x & =\int_{X} d x \int_{-1 / 2}^{x} \xi(M x) d \bar{x}-\int_{X} d x \int_{x}^{1 / 2} \xi(M x) d \bar{x} \\
& =\int_{A} \xi(M x) d x d \bar{x}+\int_{\{(x, \bar{x}) \mid x>\bar{x}, M x=M \bar{x}\}} \xi(M x) d x d \bar{x} \\
& -\int_{B} \xi(M x) d x d \bar{x}-\int_{\{(x, \bar{x}) \mid x<\bar{x}, M x=M \bar{x}\}} \xi(M x) d x d \bar{x} \\
& =\int_{A} \xi(M x) d x d \bar{x}-\int_{B} \xi(M x) d x d \bar{x} \\
& =\int_{A} \xi(M x) W(x, \bar{x}) d x d \bar{x}+\int_{B} \xi(M x) W(x, \bar{x}) d x d \bar{x} .(48)
\end{aligned}
$$

To obtain the equality in (47), we have used that

$$
\int_{\{(x, \bar{x}) \mid x>\bar{x}, M x=M \bar{x}\}} \xi(M x) d x d \bar{x}=\int_{\{(x, \bar{x}) \mid x<\bar{x}, M x=M \bar{x}\}} \xi(M x) d x d \bar{x} .
$$

By Fubini's theorem

$$
\begin{aligned}
\int_{\{M x=M \bar{x}\}} \xi(M x) W(x, \bar{x}) d x d \bar{x} & =\int_{\{M x=M \bar{x}\}} \xi(M \bar{x}) W(\bar{x}, x) d \bar{x} d x \\
& =-\int_{\{M x=M \bar{x}\}} \xi(M x) W(x, \bar{x}) d \bar{x} d x
\end{aligned}
$$

and so, the three previous expressions vanish. This, together with (48), yields the proof.

Theorem 4. Setting $\bar{H}(t):=\frac{1}{2} \int_{X}\left|\left(M^{1}\right)_{t}^{\prime}\right|^{2} d \nu_{0}+\frac{1}{2} W_{2}^{2}\left(\sigma_{t}^{1}, \nu_{0}\right)$, we have that $\bar{H}(0)=\bar{H}(t)$ for all $t \in[0, T]$. 
Proof. To simplify the notation, take $M:=M^{1}$. Let $\varphi \in C_{c}^{1}(0, T)$ be arbitrary. Since $M, M^{\prime} \in H^{1}\left(0, T ; L^{2}\left(\nu_{0}\right)\right)$, it can be shown that

$$
\lim _{h \rightarrow 0} \int_{X_{T}}\left|\frac{\left(M_{t+h} x\right)^{2}-\left(M_{t} x\right)^{2}}{h}-2 M_{t} x M_{t}^{\prime} x\right| d x d t=0
$$

and

$$
\lim _{h \rightarrow 0} \int_{X_{T}}\left|\frac{\left(M_{t+h}^{\prime} x\right)^{2}-\left(M_{t}^{\prime} x\right)^{2}}{h}-2 M_{t}^{\prime} x M_{t}^{\prime \prime} x\right| d x d t=0 .
$$

By the above limits and theorem 3, one has

$$
\begin{aligned}
-\int_{0}^{T} \varphi^{\prime}(t) \bar{H}(t) d t & =\frac{1}{2} \lim _{h \rightarrow 0} \int_{X_{T}} \frac{\varphi(t-h)-\varphi(t)}{h}\left[\left|M_{t}^{\prime}\right|^{2}+\left|M_{t}-\mathbf{i d}\right|^{2}\right] d \nu_{0} d t \\
& =\int_{X_{T}} \varphi(t) M_{t}^{\prime}\left(M_{t}^{\prime \prime}+M_{t}-\mathbf{i d}\right) d \nu_{0} d t \\
& =\int_{0}^{T} \varphi(t) \int_{X} M_{t}^{\prime} x\left(\frac{1}{2} \int_{X} W_{M}(x, \bar{x}, t) d \bar{x}-x\right) d x d t .
\end{aligned}
$$

Thus, by combining with proposition 6 , we have for $\mathcal{L}^{1}$-a.e. $t \in(0, T)$,

$$
\frac{d}{d t} \bar{H}(t)=\int_{X} \mathbf{v}_{t}\left(M_{t} x\right)\left(\frac{1}{2} \int_{X} W_{M}(x, \bar{x}, t) d \bar{x}-x\right) d x .
$$

We use lemma 10 to conclude that $\frac{d}{d t} \bar{H}(t)=0$ for $\mathcal{L}^{1}$-a.e. $t \in(0, T)$. Because $\bar{H}$ is absolutely continuous, $\bar{H}(t)=\bar{H}(0)$ for every $t \in(0, T]$.

\subsection{Minimizing paths whose endpoints are discrete measures.}

We denote by $\mathcal{P}$. $n$ the set of measures of the form $1 / n \sum_{i=1}^{n} \delta_{x_{i}}$ where $\left(x_{1}, \cdots, x_{n}\right) \in \mathbb{R}^{n}$. We show that if $\bar{\sigma}_{0}^{1}, \bar{\sigma}_{T}^{1} \in \mathcal{P}_{. n}$ and $\sigma^{1}$ minimizes $\mathcal{A}_{T}$ over $\mathcal{C}_{T}\left(\bar{\sigma}_{0}^{1}, \bar{\sigma}_{T}^{1}\right)$ then at each time $t, \sigma_{t}^{1} \in \mathcal{P}_{\text {. } n}$. Let $c_{i}=-1 / 2+i / n$ for $i=0,1, \cdots, n$ and

$$
L_{n}=\left\{S: X \rightarrow \mathbb{R}|S|_{\left(c_{i-1}, c_{i}\right)} \text { is constant for each } i=1, \cdots, n\right\},
$$

which is a closed subspace of $L^{2}\left(\nu_{0}\right)$. For $\bar{M}_{0}, \bar{M}_{T} \in L_{n}$, we denote by $\mathcal{C}_{T}^{n}\left(\bar{M}_{0}, \bar{M}_{T}\right)$ the set of paths $M \in A C^{2}\left(0, T ; L_{n}\right)$ satisfying $M_{0}=\bar{M}_{0}$ and $M_{T}=\bar{M}_{T}$. To $\mathbf{x}=\left(x_{1}, \cdots, x_{n}\right) \in \mathbb{R}^{n}$, we associate the measure $\sigma_{\mathbf{x}}=$ $1 / n \sum_{i=1}^{n} \delta_{x_{i}}$ and the map $M_{\mathbf{x}} \in L_{n}$ defined by

$$
M_{\mathbf{x}}(x)=x_{i} \quad \text { if } \quad x \in\left(c_{i-1}, c_{i}\right) .
$$

For $\mathbf{r} \in H^{1}\left(0, T ; \mathbb{R}^{n}\right)$, we define the action

$\mathcal{A}_{\cdot n}^{s}(\mathbf{r})=\frac{1}{2} \int_{0}^{T} \sum_{i=1}^{n}\left(\frac{\left|\dot{r}_{i}(t)\right|^{2}}{n}-\frac{\left|r_{i}(t)\right|^{2}}{n}\right) d t+\frac{1}{4 n s} \int_{0}^{T} \sum_{i, j=1}^{n}\left|r_{i}(t)-r_{j}(t)\right|^{s}-\frac{T}{24}$.

Note that $\mathcal{A}_{\cdot n}^{s}(\mathbf{r})=\mathcal{A}_{T}^{s}\left(\sigma_{\mathbf{r}}\right)$. 
Remark 8. It is easy to show that $\mathcal{A}_{\cdot n}^{s}$ admits a minimizer $\mathbf{r}^{s}$ over over the set $\left\{\mathbf{r} \in H^{1}(0, T)^{n} \mid \mathbf{r}(0)=\mathbf{x}^{0}, \mathbf{r}(T)=\mathbf{x}^{T}\right\}$. For $s \in(1,2)$, the EulerLagrange equations satisfied are

$$
\ddot{\mathbf{r}}_{i}^{s}(t)+\mathbf{r}_{i}^{s}(t)=\frac{s}{2 n} \sum_{j \neq i}\left(\mathbf{r}_{i}^{s}(t)-\mathbf{r}_{j}^{s}(t)\right) \mid\left(\mathbf{r}_{i}^{s}(t)-\left.\mathbf{r}_{j}^{s}(t)\right|^{s-2}, \quad i=1, \cdots, n,\right.
$$

in the sense of distributions on $(0, T)$. This proves that $\mathbf{r}^{s} \in H^{2}(0, T)^{n}$. For $\mathbf{u} \in H^{1}(0, T)^{n}$ (independent of $s$ ) and $s \in[1,2]$, we have

$$
\mathcal{A}_{\cdot n}^{s}\left(\mathbf{r}^{s}\right) \leq \sup _{\bar{s} \in[1,2]} \mathcal{A}_{\cdot n}^{\bar{s}}(\mathbf{u})<\infty
$$

and so, Poincaré's inequality gives that $\left\|\mathbf{r}^{s}\right\|_{H^{1}(0, T)^{n}}$ is bounded by a constant independent of $s$. This, together with (50) yields that $\left\|\mathbf{r}^{s}\right\|_{H^{2}(0, T)^{n}}$ is bounded by a constant independent of $s$.

We show that the set $\mathcal{P}_{\cdot n}(\mathbb{R})$ is closed under the Lagrangian minimizing paths in the following sense.

Theorem 5. Suppose $\bar{\sigma}_{0}=1 / n \sum_{i=1}^{n} \delta_{x_{i}^{0}}, \bar{\sigma}_{T}=1 / n \sum_{i=1}^{n} \delta_{x_{i}^{T}}$ and let $\sigma^{1}$ be the path found in corollary 1 , minimizing $\mathcal{A}_{T}^{1}$ over $\mathcal{C}_{T}\left(\bar{\sigma}_{0}, \bar{\sigma}_{T}\right)$. Then $\sigma_{t}^{1} \in \mathcal{P} \cdot n(\mathbb{R})$ for a.e. $t \in(0, T)$.

Proof. Assume without loss of generality that $x_{i-1}^{k} \leq x_{i}^{k}$ for $k=0, T$ and $i=1, \cdots, n$. We set

$$
\bar{M}_{0}=M_{\mathbf{x}^{0}}, \quad \bar{M}_{T}=M_{\mathbf{x}^{T}}, \quad \mathbf{x}^{k}=\left(x_{1}^{k}, \cdots, x_{n}^{k}\right) \quad \text { for } k=0, T .
$$

Let $s \in[1,2]$. We are going to apply proposition 3 with $\mathcal{S}=\left(L_{n},\|\cdot\|_{L^{2}\left(\nu_{0}\right)}\right)$ and $\tau$ is the $L^{2}$-weak topology which coincides with the $\|\cdot\|_{\nu_{0}}$-topology when restricted to $L_{n}$. Since bounded sets of $L_{n}$ are closed for the $\tau$-topology, we can apply proposition 3 and obtain as in proposition 2, existence of a path $M^{s}$ minimizing $\mathcal{Q}+\mathcal{C}^{s}$ over $A C^{2}\left(0, T ; L_{n}\right)$. Let $\sigma^{s}(t)=M_{\#}^{s} \nu_{0}$ and let $\bar{M}_{t}^{s}$ be monotone nondecreasing such that $\sigma^{s}(t)=\bar{M}_{\#}^{s} \nu_{0}$. As argued in the proof of theorem 2 , by remark 5 ,

$$
\bar{M}^{s} \in A C^{2}\left(0, T ; L_{n}\right), \quad \text { and } \quad \mathcal{Q}\left(\bar{M}^{s}\right)+\mathcal{C}^{s}\left(\bar{M}^{s}\right) \leq \mathcal{Q}\left(M^{s}\right)+\mathcal{C}^{s}\left(M^{s}\right) .
$$

The strict convexity of $\mathcal{Q}+\mathcal{C}^{s}$ (direct consequence of (30)) yields $M^{s}=$ $\bar{M}^{s}$. This proves that $M_{t}^{s}$ is monotone nondecreasing for each $t \in[0, T]$. If $\mathbf{r}=\left(r_{1}, \cdots, r_{n}\right) \in H^{1}\left(0, T ; \mathbb{R}^{n}\right)$, by abuse of notation we denote the map $t \rightarrow M_{\mathbf{r}(t)} \in L_{n}$ by $M_{\mathbf{r}}$. Set

$$
\sigma_{\mathbf{r}(t)}=\frac{1}{n} \sum_{i=1}^{n} \delta_{r_{i}(t)}, \quad r_{i}^{s}(t):=M_{t}^{s}\left(\left(c_{i-1}+c_{i}\right) / 2\right) \quad(i=1, \cdots n) .
$$

If $\mathbf{r}(0)=\mathbf{x}^{0}$ and $\mathbf{r}(T)=\mathbf{x}^{T}$ then $M_{\mathbf{r}}(0)=\bar{M}_{0}$ and $M_{\mathbf{r}}(T)=\bar{M}_{T}$. By lemma 1 and remark 1

$$
\left\|\left(M^{s}\right)^{\prime}\right\|_{m e t r i c}=\left\|\left(\sigma^{s}\right)^{\prime}\right\|_{m e t r i c}, \quad\left\|\left(M_{\mathbf{r}}\right)^{\prime}\right\|_{m e t r i c} \geq\left\|\left(\sigma_{\mathbf{r}}\right)^{\prime}\right\|_{m e t r i c} .
$$


Thus

$$
\begin{aligned}
\mathcal{A}_{\cdot n}^{s}\left(\mathbf{r}^{s}\right)=\mathcal{A}_{T}^{s}\left(\sigma^{s}\right) & =\mathcal{Q}\left(M^{s}\right)+\mathcal{C}^{s}\left(M^{s}\right)-T / 24 \\
& \leq \mathcal{Q}\left(M_{\mathbf{r}}\right)+\mathcal{C}^{s}\left(M_{\mathbf{r}}\right)-T / 24=\mathcal{A}_{T}^{s}\left(\sigma_{\mathbf{r}}\right)=\mathcal{A}_{\cdot n}^{s}(\mathbf{r})
\end{aligned}
$$

This proves that $\mathbf{r}^{s}$ minimizes $\mathcal{A}_{\cdot n}^{s}$ over the set $\left\{\mathbf{r} \in H^{1}(0, T)^{n} \mid \mathbf{r}(0)=\right.$ $\left.\mathbf{x}^{0}, \mathbf{r}(T)=\mathbf{x}^{T}\right\}$. We use remark 8 to obtain existence of a sequence $\left\{s_{j}\right\}_{j=1}^{\infty}$ converging to 1 as $j$ tends to $\infty$, such that $\left\{\mathbf{r}^{s_{j}}\right\}_{j=1}^{\infty}$ converges to some $\mathbf{r}^{1}$ in $C^{1}([0, T])$. Extracting if necessary a subsequence we do not relabel, we obtain that $\left\{w_{i j}^{s_{j}}\right\}_{j=1}^{\infty}$ converges weakly $*$ in $L^{\infty}(0, T)$ to some $w_{i j}$ (here $w_{i j}^{s}(t):=\left(\mathbf{r}_{i}^{s}(t)-\mathbf{r}_{j}^{s}(t)\right) \mid\left(\mathbf{r}_{i}^{s}(t)-\left.\mathbf{r}_{j}^{s}(t)\right|^{s-2}\right.$ from (50)). It is apparent that $w_{i j}=-w_{j i}$ and $w_{i j}(t) \in \partial .|\cdot|\left(r_{i}^{1}(t)-r_{j}^{1}(t)\right)$. One uses (50) to obtain that

$$
\ddot{\mathbf{r}}_{i}^{1}(t)+\mathbf{r}_{i}^{1}(t)=\frac{1}{2 n} \sum_{j \neq i} w_{i j}^{1}(t), \quad i=1, \cdots, n .
$$

We set $w_{i i}^{1}(t) \equiv 0$ and

$$
M(x, t)=\mathbf{r}_{i}^{1}(t), \quad W(x, \bar{x}, t)=w_{i j}^{1}(t)
$$

for $x \in\left(c_{i-1}, c_{i}\right)$ and $\bar{x} \in\left(c_{j-1}, c_{j}\right)$ to discover that $\tilde{M}_{0}^{1}=\bar{M}_{0}, M_{T}^{1}=\bar{M}_{T}$ and

$$
\left(M^{1}\right)_{t}^{\prime \prime} x+M_{t}^{1} x=\frac{1}{2} \int_{X} W(x, \bar{x}, t) d \bar{x} .
$$

This, together with theorem 3 , shows that $M^{1}$ is the unique minimizer of $\mathcal{Q}+\mathcal{C}$ over $\mathcal{C}_{T}\left(\bar{M}_{0}, \bar{M}_{T}\right)$. Set $\sigma_{t}^{1}=M_{t \#}^{1} \nu_{0} \in \mathcal{P}^{n}(\mathbb{R})$. We use corollary 1 to conclude that $\sigma^{1}$ is the unique minimizer of $\mathcal{A}_{T}$ over $\mathcal{C}_{T}\left(\bar{\sigma}_{0}, \bar{\sigma}_{T}\right)$.

Remark 9. To motivate the statement made in the introduction about the behavior of a simple two-particle system, let us consider $\mu_{0}=1 / 2\left(\delta_{x_{0}}+\delta_{y_{0}}\right)$, $\mu_{T}=1 / 2\left(\delta_{x_{T}}+\delta_{y_{T}}\right)$ and let $\sigma^{1}$ be the unique minimizer of $\mathcal{A}_{T}$ over $\mathcal{C}\left(\mu_{0}, \mu_{T}\right)$ with $T<\pi$. We learned from the closedness principle of the set $\mathcal{P}_{\text {. } 2}$ that $\sigma^{1}$ must satisfy $\sigma_{t}^{1}=1 / 2\left(\delta_{x_{t}}+\delta_{y_{t}}\right)$. Assume $t_{1}<t_{2}, x\left(t_{1}\right)=y\left(t_{1}\right)=: a_{1}$ and $x\left(t_{2}\right)=y\left(t_{2}\right)=: a_{2}$. In other words, the two particles of the system collide at two distinct times. Then, it is obvious that we must have $x(t)=y(t)$ for all $t \in\left[t_{1}, t_{2}\right]$. Indeed, let $z$ be the unique solution of the ODE $\ddot{z}+z=0$ on $\left[t_{1}, t_{2}\right]$ such that $z\left(t_{1}\right)=a_{1}$, and $z\left(t_{2}\right)=a_{2}$. Then the map $M_{t} x=z(t)$ defined on $\left(t_{1}, t_{2}\right) \times X$ and $W_{M}(y, \bar{y}, t) \equiv 0$ defined on $X^{2} \times\left(t_{1}, t_{2}\right)$ satisfies (6). Thus, it minimizes the action

$$
M \rightarrow \int_{t_{1}}^{t_{2}} d t \int_{X}\left(\left|M^{\prime}\right|^{2}-|M|^{2}\right) d x+\frac{1}{2} \int_{t_{1}}^{t_{2}} d t \int_{X^{2}}\left|M_{t} x-M_{t} \bar{x}\right| d x d \bar{x}
$$

over $\mathcal{C}\left(a_{1}, a_{2}\right)$. Define $\sigma_{t}:=M_{t \#} \nu_{0}$. Since $\sigma$ minimizes $\int_{t_{1}}^{t_{2}} L\left(\sigma_{t}, v_{t}\right) d t$ over $\mathcal{C}\left(\delta_{a_{1}}, \delta_{a_{2}}\right)$, we conclude that $\sigma_{t}=\sigma_{t}^{1}$ for $\left[t_{1}, t_{2}\right]$. 
4.5. Minimizing paths $\sigma^{1}$ such that $\sigma^{1}(0), \sigma^{1}(1)<<\mathcal{L}^{1}$.

In this subsection, we show that minimizing paths $\sigma^{1}$ may escape the set of absolutely continuous measures in spite of $\sigma^{1}(0), \sigma^{1}(1)<<\mathcal{L}^{1}$. Suppose that $T=1$ and $\bar{\sigma}_{0}<<\mathcal{L}^{1}$ and $\bar{\sigma}_{1}<<\mathcal{L}^{1}$ are two Borel probability measures. The main observation in this section is that they may be chosen so that if $\sigma^{1}$ minimizes $\mathcal{A}_{1}$ over $\mathcal{C}_{1}\left(\bar{\sigma}_{0}, \bar{\sigma}_{1}\right)$ then $\sigma_{t}^{1}=\delta_{0}$ for all $t \in[1 / 2,3 / 4]$. In other words, $\sigma^{1}$ does not entirely lie inside the set of measures that are absolutely continuous with respect to $\mathcal{L}^{1}$.

In light of theorem 3 and corollary 1 , to identify appropriate $\bar{\sigma}_{0}$ and $\bar{\sigma}_{1}$, it suffices to determine maps $M_{t}^{1}: X \rightarrow \mathbb{R}$ monotone nondecreasing which satisfy the following properties. (i) $M^{1} \in \mathcal{C}_{1}\left(\bar{M}_{0}, \bar{M}_{1}\right)$, (ii) (44) holds, (iii) $M_{0 \#}^{1} \nu_{0}, M_{1 \#}^{1} \nu_{0}<<\mathcal{L}^{1}$ and (iv) $M_{t}^{1} \equiv 0$ for all $t \in[1 / 2,3 / 4]$. Define

$$
f(t)=\frac{\cos (t-1 / 2)}{\cos 1 / 2}, \quad \alpha_{t}=1-\frac{f(t)}{f(1 / 2)}, \quad M_{t} x=\alpha_{t} x \quad t \in[0,1] .
$$

Note that $\alpha_{t}>0$ unless $t=1 / 2$ and $\alpha_{1 / 2}=0$. The map $M_{t}$ is increasing (as a function of $x$ ) for $t \in[0,1]$ provided that $t \neq 1 / 2$. But $M_{1 / 2}, M_{1 / 2}^{\prime} \equiv 0$. Since $\ddot{f}+f \equiv 0$, we have that $M_{t}^{\prime \prime} x+M_{t} x=x$ for every $(t, x) \in X_{1}$. Set $W(x, \bar{x}, t)=(x-\bar{x}) /|x-\bar{x}|$. We use that $M \in C^{\infty}\left(X_{1}\right)$ to conclude that

$$
M_{t}^{\prime \prime} x+M_{t} x=x=\frac{1}{2} \int_{X} W(x, \bar{x}, t) d \bar{x},
$$

in the sense of distributions on $X_{1}$. Similarly, we define

$$
g(t)=\frac{\cos (t-3 / 4)}{\cos 1 / 4}, \quad \beta_{t}=1-\frac{g(t)}{g(3 / 4)}, \quad N_{t} x=\beta_{t} x \quad t \in[1 / 2,1]
$$

and $\beta=\beta_{1}=1-\cos (1 / 4)$. As before, the map $N_{t}$ is increasing (as a function of $x$ ) for $t \in[1 / 2,1]$ provided that $t \neq 3 / 4$. Note that $N_{3 / 4}, M_{3 / 4}^{\prime} \equiv 0$. We use that $N \in C^{\infty}\left(X_{1}\right)$ to obtain

$$
N_{t}^{\prime \prime} x+N_{t} x=x=\frac{1}{2} \int_{X} W(x, \bar{x}, t) d \bar{x},
$$

in the sense of distributions on $(1 / 2,1) \times X$.

The monotone maps $M^{1}$. For $(x, t) \in X_{1}$, we define

$$
\begin{gathered}
M_{t}^{1} x=\left\{\begin{array}{lll}
M_{t} x & \text { if } & t \in[0,1 / 2] \\
0 & \text { if } & t \in[1 / 2,3 / 4], \\
N_{t} x & \text { if } & t \in[3 / 4,1]
\end{array}\right. \\
W_{M^{1}}(x, \bar{x}, t)=\left\{\begin{array}{lll}
\frac{x-\bar{x}}{|x-\bar{x}|} & \text { if } t \in[0,1 / 2] \\
0 & \text { if } t \in[1 / 2,3 / 4] \\
\frac{x-\bar{x}}{|x-\bar{x}|} & \text { if } t \in[3 / 4,1] .
\end{array}\right.
\end{gathered}
$$


We have that

$$
W_{M^{1}}(x, \bar{x}, t) \in \partial .|\cdot|\left(M_{t}^{1} x-M_{t}^{1} \bar{x}\right) \text { for } \mathcal{L}^{3}-\text { a.e. }(x, \bar{x}, t) \in X^{2} \times(0,1) .
$$

Clearly, $M \in C\left([0,1], L^{2}\left(\nu_{0}\right)\right)$. Since

$$
\left(M^{1}\right)_{(1 / 2)^{-}}^{\prime}=\left(M^{1}\right)_{(1 / 2)^{+}}^{\prime} \text { and }\left(M^{1}\right)_{(3 / 4)^{-}}^{\prime}=\left(M^{1}\right)_{(3 / 4)^{+}}^{\prime}
$$

we obtain that $M \in H^{2}\left(0,1 ; L^{2}\left(\nu_{0}\right)\right)$. One uses $(52),(53)$ and that

$$
\left(M^{1}\right)_{t}^{\prime \prime} x+M_{t}^{1} x=0=\frac{1}{2} \int_{X} W_{M^{1}}(x, \bar{x}, t) d \bar{x},
$$

in the sense of distributions on $(1 / 2,3 / 4) \times X$ to obtain that

$$
\left(M^{1}\right)_{t}^{\prime \prime} x+M_{t}^{1} x=\frac{1}{2} \int_{X} W_{M^{1}}(x, \bar{x}, t) d \bar{x} .
$$

in the sense of distributions on $X_{1}$.

Note that if we set $\sigma_{t}^{1}=M_{t \#}^{1} \nu_{0}$ then

$$
\sigma_{t}^{1}=\left\{\begin{array}{lll}
\frac{1}{\alpha_{t}} \chi_{\left(-\alpha_{t} / 2, \alpha_{t} / 2\right)} & \text { if } & t \in[0,1 / 2) \\
\delta_{0} & \text { if } & t \in[1 / 2,3 / 4] \\
\frac{1}{\beta_{t}} \chi_{\left(-\beta_{t} / 2, \beta_{t} / 2\right)} & \text { if } & t \in(3 / 4,1] .
\end{array}\right.
$$

The velocity in Lagrangian and Eulerian coordinates is given by

$$
\begin{gathered}
\left(M^{1}\right)_{t}^{\prime} x= \begin{cases}-\tan \left(t-\frac{1}{2}\right) x & \text { if } \quad t \in[0,1 / 2) \\
0 & \text { if } \quad t \in[1 / 2,3 / 4], \\
-\tan \left(t-\frac{3}{4}\right) x & \text { if } t \in[3 / 4,1]\end{cases} \\
v_{t}^{1}(y)=\left\{\begin{array}{lll}
-\frac{\tan \left(t-\frac{1}{2}\right)}{\alpha_{t}} y & \text { if } & t \in[0,1 / 2) \\
0 & \text { if } t \in(1 / 2,3 / 4) \\
-\frac{\tan \left(t-\frac{3}{4}\right)}{\beta_{t}} y & \text { if } & t \in(3 / 4,1) .
\end{array}\right.
\end{gathered}
$$

We have proven the following theorem.

Theorem 6. Let

$$
\begin{gathered}
\bar{\sigma}_{0}=\frac{1}{\alpha_{0}} \chi_{\left(-\alpha_{0} / 2, \alpha_{0} / 2\right)}, \quad \bar{\sigma}_{1}=\frac{1}{\beta_{1}} \chi_{\left(-\beta_{1} / 2, \beta_{1} / 2\right)}, \\
v_{0}(y)=\frac{\tan 1 / 2}{1-\cos 1 / 2} \text { y for } \quad|y| \leq \alpha_{0} / 2 .
\end{gathered}
$$

(i) Optimal path escaping the set of absolutely continuous measures. $\sigma^{1}$, defined above, minimizes $\mathcal{A}_{1}^{1}$ over $\mathcal{C}_{1}\left(\bar{\sigma}_{0}, \bar{\sigma}_{1}\right)$ and $\sigma_{t}^{1}=\delta_{0}$ for $t \in[1 / 2,3 / 4]$.

(ii) Non uniqueness in the initial value problem. The path $\sigma$ defined by $\sigma_{t}=M_{t \# \nu_{0}}$, minimizes $\mathcal{A}_{1}^{1}$ over $\mathcal{C}_{1}\left(\bar{\sigma}_{0}, \bar{\sigma}_{0}\right)$. Thus, $\sigma$ and $\sigma^{1}$ are two solutions of the Euler-Poisson system, distinct for $t \in(1 / 2,3 / 4)$, with the same initial point $\bar{\sigma}_{0}$ and the same initial velocity $v_{0}=v_{0}^{1}$. 


\section{References}

1. L. Ambrosio, W. Gangbo: Hamiltonian ODE in the Wasserstein spaces of probability measures. To appear in Comm. Pure Appl. Math.

2. L. Ambrosio, N. Gigli, G. Savaré: Gradient flows in metric spaces and the Wasserstein spaces of probability measures. Lectures in Mathematics, ETH Zurich, Birkhäuser, 2005.

3. L. Ambrosio, F. Santambrogio: Necessary optimality conditions for geodesics in weighted Wasserstein spaces. (preprint).

4. F. Bolley, Y. Brenier, G. Loeper: Contractive metrics for scalar conservation laws. J. Hyperbolic Differ. Eq. 2, no. 1, 91-107 (2005).

5. F. Bouchut: Renormalized solutions to the Vlasov equation with coefficients of bounded variation. Arch. Rational Mech. Anal., 157 75-90 (2001).

6. Y. BRENIER: Order preserving vibrating strings and applications to Electrodynamics and Magnetohydrodynamics. Methods Appl. Anal. 11, no 4, 515-532 (2004).

7. J.D. Benamou, Y. Brenier: Weak existence for the semigeostrophic equations formulated as a coupled Monge-Ampère equations/transport problem. SIAM J. Appl. Anal. Math. 58, no 5, 1450-1461 (1998).

8. Y. Brenier, E. Grenier: Sticky particles and scalar conservation laws. SIAM J. Numer. Anal. 35, no 6, 2317-2328 (1998).

9. Y. Brenier, G. LoePer: A geometric approximation to the Euler equations: The Vlasov-Monge-Ampère equation. Geom. Funct. Anal., (2004).

10. J. A. Carrillo, M. Di Francesco, C. Lattanzio: Contractivity of Wasserstein Metrics and Asymptotic Profiles for Scalar Conservation Laws. Preprint.

11. S. Cordier, P. Degond, P. Markowich, C. Schmeiser: Travelling wave analysis of an isothermal Euler-Poisson model. Annales de la Faculté des Sciences de Toulouse, vol V, no 4, 598-643 (1996).

12. M. Cullen, W. Gangbo, G. Pisante: Semigeostrophic equations discretized in reference and dual variables. To appear Arch. Rat. Mech. Analysis.

13. W. E, Y. RYkov, Y. SinAI: Generalized variational principles, global weak solutions and behavior with random initial data for systems of conservation laws arising in adhesion particle dynamics. Comm. Math. Phys. 177, 349-380 (1996).

14. W. Gangbo, R.J. McCann: The geometry of optimal transportation. Acta Math. 177, 113-161 (1996).

15. B.J. Hoskins: The geostrophic momentum approximation and the semigeostrophic equations. J. Atmosph. Sciences 32, 233-242 (1975).

16. F. Huang, Z. Wang: Well Posedness for Pressureless Flow. Comm. Math. Phys. 222, 117-146 (2001).

17. P. Markowich, C. Ringhofer, C. Schmeiser: Semiconductor Equations. Springer-Verlag (1990).

18. R. J. MCCANN: A convexity principle for interacting gases. Adv. Math. 128, no. 1, 153-179 (1997).

19. G. Monge: Mémoire sur la théorie des déblais et de remblais. Histoire de l'Académie Royale des Sciences de Paris, avec les Mémoires de Mathématique et de Physique pour la même année, 666-704 (1781).

20. Y.J. Peng: Convergence of the fractional step Lax-Friedrichs scheme and Godunov scheme for a nonlinear Euler-Poisson system. Nonlin. Anal. 42, 1033-1054 (2000).

21. C. Villani: Topics in optimal transportation. Graduate Studies in Mathematics 58, American Mathematical Society, 2003. 
W. Gangbo

School of Mathematics

Georgia Institute of Technology

Atlanta, GA 30332

email:gangbo@math.gatech.edu

T. Nguyen

Department of Theoretical and Applied Mathematics

University of Akron

Akron, OH 44325

email:tn8@uakron.edu

and

A. Tudorascu

School of Mathematics

Georgia Institute of Technology

Atlanta, GA 30332

email:adriant@math.gatech.edu 